\title{
Hybrid implicit steepest-descent methods for triple hierarchical variational inequalities with hierarchical variational inequality constraints
}

\author{
Lu-Chuan Ceng ${ }^{a}$, Yeong-Cheng Liou ${ }^{b, c, *}$, Ching-Feng Wen ${ }^{d}$, Ching-Hua Lo ${ }^{e, *}$ \\ a Department of Mathematics, Shanghai Normal University, and Scientific Computing Key Laboratory of Shanghai Universities, \\ Shanghai 200234, China. \\ ${ }^{b}$ Department of Healthcare Administration and Medical Informatics, and Research Center of Nonlinear Analysis and Optimization, \\ Kaohsiung Medical University, Kaohsiung 807, Taiwan. \\ ${ }^{c}$ Department of Medical Research, Kaohsiung Medical University Hospital, Kaohsiung 807, Taiwan. \\ ${ }^{d}$ Center for Fundamental Science, Kaohsiung Medical University, Kaohsiung 80708, Taiwan. \\ ${ }^{e}$ Department of Management, Yango University, Fujian 350015, China.
}

Communicated by Y. H. Yao

\begin{abstract}
In this paper, we introduce and analyze a hybrid implicit steepest-descent algorithm for solving the triple hierarchical variational inequality problem with the hierarchical variational inequality constraint for finitely many nonexpansive mappings in a real Hilbert space. The proposed algorithm is based on Korpelevich's extragradient method, hybrid steepest-descent method, Mann's implicit iteration method, and Halpern's iteration method. Under mild conditions, the strong convergence of the iteration sequences generated by the algorithm is established. Our results improve and extend the corresponding results in the earlier and recent literature. (c)2017 All rights reserved.
\end{abstract}

Keywords: Hybrid implicit steepest-descent algorithm, triple hierarchical variational inequality, Mann's implicit iteration method, nonexpansive mapping, inverse-strong monotonicity, global convergence.

2010 MSC: 49J30, 47H09, 47J20, 49M05.

\section{Introduction}

Let $\mathrm{H}$ be a real Hilbert space with inner product $\langle\cdot, \cdot\rangle$ and norm $\|\cdot\|$. Let $\mathrm{C}$ be a nonempty closed convex subset of $H$ and $P_{C}$ be the metric projection of $H$ onto $C$. If $\left\{x_{k}\right\}$ is a sequence in $H$, then we denote by $x_{k} \rightarrow x$ (respectively, $x_{k} \rightarrow x$ ) the strong (respectively, weak) convergence of the sequence $\left\{x_{k}\right\}$ to $x$. Let $\mathrm{S}: \mathrm{C} \rightarrow \mathrm{H}$ be a nonlinear mapping on $\mathrm{C}$. We denote by $\operatorname{Fix}(\mathrm{S})$ the set of fixed points of $\mathrm{S}$ and by $\mathbf{R}$ the set of all real numbers. A mapping $\mathrm{S}: \mathrm{C} \rightarrow \mathrm{H}$ is called L-Lipschitz continuous if there exists a constant $\mathrm{L} \geqslant 0$ such that

$$
\|S x-S y\| \leqslant L\|x-y\|, \quad \forall x, y \in C
$$

\footnotetext{
*Corresponding authors

Email addresses: zenglc@hotmail.com (Lu-Chuan Ceng), simplex_liou@hotmail.com (Yeong-Cheng Liou), cf wen@kmu.edu.tw (Ching-Feng Wen), bde_lo@sina.com (Ching-Hua Lo)
}

doi:10.22436/jnsa.010.07.47 
In particular, if $L=1$ then $S$ is called a nonexpansive mapping; if $L \in[0,1)$ then $S$ is called a contraction.

Let $\mathcal{A}: \mathrm{C} \rightarrow \mathrm{H}$ be a nonlinear mapping on $\mathrm{C}$. The classical variational inequality problem (VIP) is to find $x \in \mathrm{C}$ such that

$$
\langle\mathcal{A} x, y-x\rangle \geqslant 0, \quad \forall y \in C .
$$

The solution set of VIP $(1.1)$ is denoted by $\operatorname{VI}(C, \mathcal{A})$. In 1976, Korpelevich [13] proposed an iterative algorithm for solving the VIP (1.1) in Euclidean space $\mathbf{R}^{\mathrm{n}}$ :

$$
\left\{\begin{array}{l}
y_{k}=P_{C}\left(x_{k}-\tau A x_{k}\right), \\
x_{k+1}=P_{C}\left(x_{k}-\tau A y_{k}\right), \quad \forall k \geqslant 0,
\end{array}\right.
$$

with $\tau>0$ a given number, which is known as the extragradient method. The literature on the VIP is vast and Korpelevich's extragradient method has received great attention given by many authors, who improved it in various ways; see e.g., $[1,5,6,24-26]$ and references therein.

Let $\mathrm{A}: \mathrm{C} \rightarrow \mathrm{H}$ and $\mathrm{B}: \mathrm{H} \rightarrow \mathrm{H}$ be two mappings. Consider the following bilevel variational inequality problem (BVIP).

Problem 1.1. We find $x^{*} \in \mathrm{VI}(\mathrm{C}, \mathrm{B})$ such that

$$
\left\langle A x^{*}, x-x^{*}\right\rangle \geqslant 0, \quad \forall x \in \operatorname{VI}(C, B),
$$

where $\operatorname{VI}(C, B)$ denotes the set of solutions of the VIP: find $y^{*} \in C$ such that

$$
\left\langle B y^{*}, y-y^{*}\right\rangle \geqslant 0, \quad \forall y \in C .
$$

Bilevel variational inequalities are special classes of quasivariational inequalities ([7]) and of equilibrium with equilibrium constraints considered in $[14,16]$. However it covers some classes of mathematical programs with equilibrium constraints ([14]), bilevel minimization problems ([17]), variational inequalities ([27]), and complementarity problems.

In what follows, suppose that $A$ and $B$ satisfy the following conditions:

(C1) $B$ is pseudomonotone on $\mathrm{H}$ and $A$ is $\beta$-strongly monotone on $C$;

(C2) $A$ is $L_{1}$-Lipschitz continuous on $C$;

(C3) $\mathrm{B}$ is $\mathrm{L}_{2}$-Lipschitz continuous on $\mathrm{H}$;

(C4) $\operatorname{VI}(C, B) \neq \emptyset$.

In 2012, Anh et al. [1] introduced the following extragradient iterative algorithm for solving the above bilevel variational inequality.

Algorithm 1.2 ([1, Algorithm 2.1]). Initialization: choose $u \in \mathbf{R}^{\mathfrak{n}}, x_{0} \in C$.

Step 1. Compute $y_{k}:=P_{C}\left(x_{k}-\lambda_{k} B x_{k}\right)$ and $z_{k}:=P_{C}\left(x_{k}-\lambda_{k} B y_{k}\right)$.

Step 2. Inner loop $j=0,1, \ldots$. Compute

$$
\left\{\begin{array}{l}
x_{k, 0}:=z_{k}-\lambda A z_{k}, \\
y_{k, j}:=P_{C}\left(x_{k, j}-\delta_{j} B x_{k, j}\right), \\
x_{k, j+1}:=\alpha_{j} x_{k, 0}+\beta_{j} x_{k, j}+\gamma_{j} P_{C}\left(x_{k, j}-\delta_{j} B y_{k, j}\right) . \\
\text { If }\left\|x_{k, j+1}-P_{V I}(C, B) x_{k, 0}\right\| \leqslant \bar{\epsilon}_{k} \text { then set } h_{k}:=x_{k, j+1} \text { and go to Step } 3 . \\
\text { otherwise, increase } j \text { by } 1 \text { and repeat the inner loop Step } 2 .
\end{array}\right.
$$

Step 3. Set $x_{k+1}:=\alpha_{k} u+\beta_{k} x_{k}+\gamma_{k} h_{k}$. Then increase $k$ by 1 and go to Step 1 .

Theorem 1.3 ([1, Theorem 3.1]). Suppose that the assumptions (C1)-(C4) hold. Then the two sequences $\left\{x_{k}\right\}$ and $\left\{z_{\mathrm{n}}\right\}$ in Algorithm 1.2 converge to the same point $x^{*}$ which is a solution of the BVIP. 
On the other hand, recall the variational inequality for a monotone operator $A_{1}: H \rightarrow H$ over the fixed point set of a nonexpansive mapping $\mathrm{T}: \mathrm{H} \rightarrow \mathrm{H}$ :

Find $\quad \bar{x} \in \operatorname{VI}\left(\operatorname{Fix}(T), A_{1}\right):=\left\{\bar{x} \in \operatorname{Fix}(T):\left\langle A_{1} \bar{x}, y-\bar{x}\right\rangle \geqslant 0, \forall y \in \operatorname{Fix}(T)\right\}$,

where $\operatorname{Fix}(T):=\{x \in H: T x=x\} \neq \emptyset$. In $[10,11]$, liduka introduced the following three-stage variational inequality problem, that is, the following monotone variational inequality with variational inequality constraint over the fixed point set of a nonexpansive mapping.

Problem 1.4 ([11, Problem 3.1]). Assume that

(i) $\mathrm{T}: \mathrm{H} \rightarrow \mathrm{H}$ is a nonexpansive mapping with $\operatorname{Fix}(\mathrm{T}) \neq \emptyset$;

(ii) $A_{1}: H \rightarrow H$ is $\alpha$-inverse strongly monotone;

(iii) $A_{2}: H \rightarrow H$ is $\beta$-strongly monotone and L-Lipschitz continuous;

(iv) $\operatorname{VI}\left(\operatorname{Fix}(T), A_{1}\right) \neq \emptyset$.

Then the objective is to find

$$
x^{*} \in \operatorname{VI}\left(\operatorname{VI}\left(\operatorname{Fix}(T), A_{1}\right), A_{2}\right)=\left\{x^{*} \in \operatorname{VI}\left(\operatorname{Fix}(T), A_{1}\right):\left\langle A_{2} x^{*}, v-x^{*}\right\rangle \geqslant 0, \forall v \in \operatorname{VI}\left(\operatorname{Fix}(T), A_{1}\right)\right\} .
$$

Since this problem has a triple structure in contrast with bilevel programming problems ([14, 16]) or hierarchical constrained optimization problems or hierarchical fixed point problem, it is referred to as a triple-hierarchical constrained optimization problem (THCOP). More precisely, it is referred as a triple hierarchical variational inequality problem (THVIP); see Ceng et al. [4]. Very recently, some authors continued the study of Iiduka's THVIP (i.e., Problem 1.4 and its variant and extension; see e.g., [2, 4, 28]).

Algorithm 1.5 ([11, Algorithm 4.1]). Let $\mathrm{T}: \mathrm{H} \rightarrow \mathrm{H}$ and $A_{i}: H \rightarrow H(i=1,2)$ satisfy the assumptions (i)-(iv) in Problem 1.4. The following steps are presented for solving Problem 1.4.

Step 0. Take $\left\{\alpha_{k}\right\}_{k=0}^{\infty},\left\{\lambda_{k}\right\}_{k=0}^{\infty} \subset(0, \infty)$, and $\mu>0$, choose $x_{0} \in H$ arbitrarily, and let $k:=0$.

Step 1. Given $x_{k} \in H$, compute $x_{k+1} \in H$ as $y_{k}:=T\left(x_{k}-\lambda_{k} A_{1} x_{k}\right)$ and $x_{k+1}:=y_{k}-\mu \alpha_{k} A_{2} y_{k}$.

Update $k:=k+1$ and go to Step 1 .

The convergence analysis of the proposed algorithm was also studied in [11]. The following strong convergence theorem is established for Algorithm 1.5.

Theorem 1.6 ([11, Theorem 4.1]). Assume that $\left\{y_{k}\right\}_{k=0}^{\infty}$ in Algorithm 1.5 is bounded. Then the sequence $\left\{x_{k}\right\}_{k=0}^{\infty}$ generated by Algorithm 1.5 satisfies the following properties.

(a) $\left\{x_{k}\right\}_{k=0}^{\infty}$ is bounded;

(b) $\lim _{k \rightarrow \infty}\left\|x_{k}-y_{k}\right\|=0$ and $\lim _{k \rightarrow \infty}\left\|x_{k}-T x_{k}\right\|=0$ hold;

(c) if $\left\|x_{k}-y_{k}\right\|=o\left(\lambda_{k}\right),\left\{x_{k}\right\}_{k=0}^{\infty}$ converges strongly to the unique solution of Problem 1.4.

In 2012, inspired by Iiduka's Algorithm 1.5, Zeng et al. [28] proposed one relaxed hybrid steepestdescent algorithm for solving Problem 1.4.

Algorithm 1.7 ([28, Algorithm 3.1]). Assume that the operators $T: H \rightarrow H$ and $A_{i}: H \rightarrow H \quad(i=1,2)$ satisfy conditions (i)-(iv) in Problem 1.4.

Step 0. Take $\left\{\alpha_{k}\right\}_{k=0}^{\infty} \subset(0,1],\left\{\lambda_{k}\right\}_{k=0}^{\infty} \subset(0,2 \alpha], \mu \in\left(0, \frac{2 \beta}{L^{2}}\right)$, choose $x_{0} \in H$ arbitrarily, and let $k:=0$.

Step 1. Given $x_{k} \in H$, compute $x_{k+1} \in H$ as $y_{k}:=T\left(x_{k}-\lambda_{k} A_{1} x_{k}\right)$ and $x_{k+1}:=y_{k}-\mu \alpha_{k} A_{2} y_{k}$.

Update $k:=k+1$ and go to Step 1 .

The following theorem provides the strong convergence criteria for Algorithm 1.7.

Theorem 1.8 ([28, Theorem 3.1]). The sequence $\left\{x_{k}\right\}_{k=0}^{\infty}$ generated by Algorithm 1.7 satisfies the following properties: 
(a) $\left\{x_{\mathrm{k}}\right\}_{\mathrm{k}=0}^{\infty}$ is bounded;

(b) $\lim _{k \rightarrow \infty}\left\|x_{k}-y_{k}\right\|=0$ and $\lim _{k \rightarrow \infty}\left\|x_{k}-T x_{k}\right\|=0$;

(c) $\left\{x_{k}\right\}_{k=0}^{\infty}$ converges strongly to the unique solution of Problem I provided $\left\|x_{k}-y_{k}\right\|=o\left(\lambda_{k}\right)$.

Moreover, the authors [28] also considered the following monotone variational inequality with the variational inequality constraint over the intersection of the fixed point sets of $\mathrm{N}$ nonexpansive mappings $\mathrm{T}_{i}: \mathrm{H} \rightarrow \mathrm{H}$, with $\mathrm{N} \geqslant 1$ an integer.

Problem 1.9 ([28, Problem II]). Assume that

(i) each $T_{i}: H \rightarrow H$ is a nonexpansive mapping with $\cap_{i=1}^{N} \operatorname{Fix}\left(T_{i}\right) \neq \emptyset$;

(ii) $A_{1}: H \rightarrow H$ is $\alpha$-inverse strongly monotone;

(iii) $A_{2}: H \rightarrow H$ is $\beta$-strongly monotone and L-Lipschitz continuous;

(iv) $\operatorname{VI}\left(\cap_{i=1}^{N} \operatorname{Fix}\left(T_{i}\right), A_{1}\right) \neq \emptyset$.

Then the objective is to find

$$
x^{*} \in \operatorname{VI}\left(\operatorname{VI}\left(\bigcap_{i=1}^{N} \operatorname{Fix}\left(T_{i}\right), A_{1}\right), A_{2}\right)=\left\{x^{*} \in \operatorname{VI}\left(\bigcap_{i=1}^{N} \operatorname{Fix}\left(T_{i}\right), A_{1}\right):\left\langle A_{2} x^{*}, v-x^{*}\right\rangle \geqslant 0, \forall v \in \operatorname{VI}\left(\bigcap_{i=1}^{N} \operatorname{Fix}\left(T_{i}\right), A_{1}\right)\right\} .
$$

Obviously, Problem 1.9 of finding a unique element of $\operatorname{VI}\left(\operatorname{VI}\left(\cap_{i=1}^{N} \operatorname{Fix}\left(T_{i}\right), A_{1}\right), A_{2}\right)$ is more general and more complex than Problem 1.4. We write $T_{[k]}:=T_{\text {kmodN }}$ for integer $k \geqslant 1$ with the mod function taking values in the set $\{1,2, \ldots, N\}$, that is, if $k=j N+q$ for some integers $j \geqslant 0$ and $0 \leqslant q<N$, then $\mathrm{T}_{[\mathrm{k}]}=\mathrm{T}_{\mathrm{N}}$ if $\mathrm{q}=0$ and $\mathrm{T}_{[\mathrm{k}]}=\mathrm{T}_{\mathrm{q}}$ if $0<\mathrm{q}<\mathrm{N}$. The authors [28] also proposed another relaxed hybrid steepest-descent algorithm for solving Problem 1.9, and established the strong convergence result for the proposed algorithm.

Algorithm 1.10 ([28, Algorithm 3.2]). Assume that the operators $T_{i}: H \rightarrow H \quad(i=1, \ldots, N)$ and $A_{j}: H \rightarrow$ $\mathrm{H}(\boldsymbol{j}=1,2)$ satisfy assumptions (i)-(iv) in Problem 1.9.

Step 0. Take $\left\{\alpha_{k}\right\}_{k=0}^{\infty} \subset(0,1],\left\{\lambda_{k}\right\}_{k=0}^{\infty} \subset(0,2 \alpha], \mu \in\left(0, \frac{2 \beta}{L^{2}}\right)$, choose $x_{0} \in H$ arbitrarily and let $k:=0$.

Step 1. Given $x_{k} \in H$, compute $x_{k+1} \in H$ as $y_{k}:=T_{[k+1]}\left(x_{k}-\lambda_{k} A_{1} x_{k}\right)$ and $x_{k+1}:=y_{k}-\mu \alpha_{k} A_{2} y_{k}$.

Update $k:=k+1$ and go to Step 1 .

Theorem 1.11 ([28, Theorem 3.2]). Assume that $\bigcap_{i=1}^{N} \operatorname{Fix}\left(T_{i}\right)=\operatorname{Fix}\left(T_{1} T_{2} \cdots T_{N}\right)=\operatorname{Fix}\left(T_{N} T_{1} \cdots T_{N-1}\right)=$ $\cdots=\operatorname{Fix}\left(\mathrm{T}_{2} \mathrm{~T}_{3} \cdots \mathrm{T}_{\mathrm{N}} \mathrm{T}_{1}\right)$. Then the sequence $\left\{\mathrm{x}_{\mathrm{k}}\right\}_{\mathrm{k}=0}^{\infty}$ generated by Algorithm 1.10 satisfies the following properties:

(a) $\left\{x_{\mathrm{k}}\right\}_{\mathrm{k}=0}^{\infty}$ is bounded;

(b) $\lim _{k \rightarrow \infty}\left\|x_{k+N}-x_{k}\right\|=0$ and $\lim _{k \rightarrow \infty}\left\|x_{k}-T_{[k+N]} \cdots T_{[k+1]} x_{k}\right\|=0$;

(c) $\left\{x_{k}\right\}_{k=0}^{\infty}$ converges strongly to the unique solution of Problem II provided $\left\|x_{k}-y_{k}\right\|=o\left(\lambda_{k}\right)$.

In this paper, we introduce and analyze a hybrid implicit steepest-descent algorithm for solving the triple hierarchical variational inequality problem (THVIP) with the hierarchical variational inequality constraint for finitely many nonexpansive mappings in a real Hilbert space. The proposed algorithm is based on Korpelevich's extragradient method [13], hybrid steepest descent method [22], Mann's implicit iteration method [29] and Halpern's iteration method. Under mild conditions, the strong convergence of the iteration sequences generated by the algorithm is derived. Our results improve and extend the corresponding results announced by some others, e.g., Iiduka [11, Theorem 4.1], Zeng et al. [28, Theorem 3.2], and Anh et al. [1, Theorem 3.1].

\section{Preliminaries}

Throughout this paper, we assume that $C$ is a nonempty closed convex subset of a real Hilbert space $H$. We use $\omega_{w}\left(x_{k}\right)$ to denote the weak $\omega$-limit set of the sequence $\left\{x_{k}\right\}$, i.e.,

$$
\omega_{w}\left(x_{k}\right):=\left\{x \in H: x_{k_{i}} \rightarrow x \text { for some subsequences }\left\{x_{k_{i}}\right\} \text { of }\left\{x_{k}\right\}\right\} .
$$

Recall that a mapping $\mathrm{A}: \mathrm{C} \rightarrow \mathrm{H}$ is called: 
(i) monotone if

$$
\langle A x-A y, x-y\rangle \geqslant 0, \quad \forall x, y \in C ;
$$

(ii) $\eta$-strongly monotone if there exists a constant $\eta>0$ such that

$$
\langle A x-A y, x-y\rangle \geqslant \eta\|x-y\|^{2}, \quad \forall x, y \in C ;
$$

(iii) $\alpha$-inverse-strongly monotone if there exists a constant $\alpha>0$ such that

$$
\langle A x-A y, x-y\rangle \geqslant \alpha\|A x-A y\|^{2}, \quad \forall x, y \in C .
$$

It is obvious that if $A$ is $\alpha$-inverse-strongly monotone, then $A$ is monotone and $\frac{1}{\alpha}$-Lipschitz continuous.

The metric (or nearest point) projection from $\mathrm{H}$ onto $\mathrm{C}$ is the mapping $\mathrm{P}_{\mathrm{C}}: \mathrm{H} \rightarrow \mathrm{C}$ which assigns to each point $x \in H$ the unique point $P_{C} x \in C$ satisfying the property

$$
\left\|x-P_{C} x\right\|=\inf _{y \in C}\|x-y\|=: d(x, C) .
$$

Some important properties of projections are gathered in the following proposition.

Proposition 2.1 ([23]). For given $\mathrm{x} \in \mathrm{H}$ and $z \in \mathrm{C}$ :

(i) $z=\mathrm{P}_{\mathrm{C}} \mathrm{x} \Leftrightarrow\langle x-z, y-z\rangle \leqslant 0, \quad \forall y \in \mathrm{C}$;

(ii) $z=\mathrm{P}_{\mathrm{C}} \mathrm{x} \Leftrightarrow\|x-z\|^{2} \leqslant\|x-y\|^{2}-\|y-z\|^{2}, \quad \forall y \in C$;

(iii) $\left\langle P_{C} x-P_{C} y, x-y\right\rangle \geqslant\left\|P_{C} x-P_{C} y\right\|^{2}, \quad \forall y \in H$.

Consequently, $\mathrm{P}_{\mathrm{C}}$ is nonexpansive and monotone.

If $A$ is an $\alpha$-inverse-strongly monotone mapping of $C$ into $H$, then it is obvious that $A$ is $\frac{1}{\alpha}$-Lipschitz continuous. We also have that, for all $u, v \in C$ and $\lambda>0$,

$$
\|(I-\lambda A) u-(I-\lambda A) v\|^{2} \leqslant\|u-v\|^{2}+\lambda(\lambda-2 \alpha)\|A u-A v\|^{2} .
$$

So, if $\lambda \leqslant 2 \alpha$, then $I-\lambda A$ is a nonexpansive mapping from $C$ to $H$.

Definition 2.2. A mapping $\mathrm{T}: \mathrm{H} \rightarrow \mathrm{H}$ is said to be:

(a) nonexpansive if $\|T x-T y\| \leqslant\|x-y\|$ for all $x, y \in H$;

(b) firmly nonexpansive if $2 \mathrm{~T}-\mathrm{I}$ is nonexpansive, or equivalently, if $\mathrm{T}$ is 1-inverse strongly monotone (1-ism),

$$
\langle x-y, T x-T y\rangle \geqslant\|T x-T y\|^{2}, \quad \forall x, y \in H ;
$$

alternatively, $\mathrm{T}$ is firmly nonexpansive if and only if $\mathrm{T}$ can be expressed as $\mathrm{T}=\frac{1}{2}(\mathrm{I}+\mathrm{S})$ where $\mathrm{S}: \mathrm{H} \rightarrow \mathrm{H}$ is nonexpansive; projections are firmly nonexpansive.

It can be easily seen that if $T$ is nonexpansive, then $\mathrm{I}-\mathrm{T}$ is monotone. It is also easy to see that a projection $\mathrm{P}_{C}$ is 1-ism. Inverse strongly monotone (also referred to as co-coercive) operators have been applied widely in solving practical problems in various fields.

Proposition 2.3. Let $\mathrm{T}: \mathrm{C} \rightarrow \mathrm{C}$ be a nonexpansive mapping. Then the following hold:

(i) Fix (T) is closed and convex ([9, Proposition 5.3]);

(ii) $\operatorname{Fix}(\mathrm{T}) \neq \emptyset$ when $\mathrm{C}$ is bounded ([9, Theorem 5.1]).

We need some facts and tools in a real Hilbert space $\mathrm{H}$ which are listed as lemmas below.

Lemma 2.4. Let $X$ be a real inner product space. Then there holds the following inequality

$$
\|x+y\|^{2} \leqslant\|x\|^{2}+2\langle y, x+y\rangle, \quad \forall x, y \in X .
$$


Recall that, a mapping $A: C \rightarrow H$ is called hemicontinuous if for all $x, y \in C$, the mapping $g:[0,1] \rightarrow$ $H$, defined by $g(t):=A(t x+(1-t) y)$, is continuous. Some properties of the solution set of the monotone variational inequality are mentioned in the following result.

Lemma 2.5. Let $\mathrm{A}: \mathrm{C} \rightarrow \mathrm{H}$ be a monotone and hemicontinuous mapping. Then the following hold:

(i) $\operatorname{VI}(C, A)$ is equivalent to $\operatorname{MVI}(C, A):=\left\{x^{*} \in C:\left\langle A y, y-x^{*}\right\rangle \geqslant 0, \forall y \in C\right\}$ ([12]);

(ii) $\operatorname{VI}(C, A) \neq \emptyset$ when $\mathrm{C}$ is bounded ([12]);

(iii) $\operatorname{VI}(C, A)=\operatorname{Fix}\left(\mathrm{P}_{C}(\mathrm{I}-\lambda \mathrm{A})\right.$ ) for all $\lambda>0$, where $\mathrm{I}$ is the identity mapping on $\mathrm{H}$ ([19, Lemma 2.24]);

(iv) $\operatorname{VI}(C, A)$ consists of only one point, if $A$ is strongly monotone and Lipschitz continuous ([19, Theorem 2.31]).

Lemma 2.6 ([8, Demiclosedness principle]). Let C be a nonempty closed convex subset of a real Hilbert space $\mathrm{H}$. Let $\mathrm{S}$ be a nonexpansive self-mapping on $\mathrm{C}$ with $\operatorname{Fix}(\mathrm{S}) \neq \emptyset$. Then $\mathrm{I}-\mathrm{S}$ is demiclosed. That is, whenever $\left\{\mathrm{x}_{\mathrm{k}}\right\}$ is a sequence in $\mathrm{C}$ weakly converging to some $\mathrm{x} \in \mathrm{C}$ and the sequence $\left\{(\mathrm{I}-\mathrm{S}) \mathrm{x}_{\mathrm{k}}\right\}$ strongly converges to some $\mathrm{y}$, it follows that $(\mathrm{I}-\mathrm{S}) \mathrm{x}=\mathrm{y}$. Here $\mathrm{I}$ is the identity operator of $\mathrm{H}$.

Recall that, a mapping $\mathrm{T}: \mathrm{C} \rightarrow \mathrm{C}$ is called a $\zeta$-strictly pseudocontractive mapping (or a $\zeta$-strict pseudocontraction) if there exists a constant $\zeta \in[0,1)$ such that

$$
\|\mathrm{T} x-\mathrm{T} y\|^{2} \leqslant\|x-y\|^{2}+\zeta\|(\mathrm{I}-\mathrm{T}) x-(\mathrm{I}-\mathrm{T}) \mathrm{y}\|^{2}, \quad \forall x, y \in \mathrm{C} .
$$

Note that the class of strictly pseudocontractive mappings strictly includes the class of nonexpansive mappings. It is clear that $\mathrm{T}$ is nonexpansive if and only if $\mathrm{T}$ is a 0 -strict pseudocontraction.

Lemma 2.7 ([15, Proposition 2.1]). Let $\mathrm{C}$ be a nonempty closed convex subset of a real Hilbert space $\mathrm{H}$ and $\mathrm{T}: \mathrm{C} \rightarrow \mathrm{C}$ be a mapping.

(i) If $\mathrm{T}$ is a -strictly pseudocontractive mapping, then $\mathrm{T}$ satisfies the Lipschitzian condition

$$
\|T x-T y\| \leqslant \frac{1+\zeta}{1-\zeta}\|x-y\|, \quad \forall x, y \in C .
$$

(ii) If $\mathrm{T}$ is a $\zeta$-strictly pseudocontractive mapping, then the mapping $\mathrm{I}-\mathrm{T}$ is semiclosed at 0 , that is, if $\left\{\mathrm{x}_{\mathrm{n}}\right\}$ is a sequence in $C$ such that $x_{n} \rightarrow \tilde{x}$ and $(I-T) x_{n} \rightarrow 0$, then $(I-T) \tilde{x}=0$.

(iii) If $\mathrm{T}$ is $\zeta$-(quasi-)strict pseudocontraction, then the fixed-point set $\operatorname{Fix}(\mathrm{T})$ of $\mathrm{T}$ is closed and convex so that the projection $\mathrm{P}_{\mathrm{Fix}(\mathrm{T})}$ is well-defined.

Lemma 2.8 ([24]). Let C be a nonempty closed convex subset of a real Hilbert space $\mathrm{H}$. Let $\mathrm{T}: \mathrm{C} \rightarrow \mathrm{C}$ be a $\zeta$-strictly pseudocontractive mapping. Let $\gamma$ and $\delta$ be two nonnegative real numbers such that $(\gamma+\delta) \zeta \leqslant \gamma$. Then

$$
\|\gamma(x-y)+\delta(T x-T y)\| \leqslant(\gamma+\delta)\|x-y\|, \quad \forall x, y \in C .
$$

The following lemma can be easily proven, and therefore, we omit the proof.

Lemma 2.9 ([3, Lemma 2.8]). Let $\left\{a_{k}\right\}_{k=0}^{\infty}$ be a bounded sequence of nonnegative real numbers and $\left\{b_{k}\right\}_{k=0}^{\infty}$ be a sequence of real numbers such that $\lim \sup _{k \rightarrow \infty} b_{k} \leqslant 0$. Then, $\lim _{\sup _{k \rightarrow \infty}} a_{k} b_{k} \leqslant 0$.

Let $\mathrm{C}$ be a nonempty closed convex subset of a real Hilbert space $\mathrm{H}$. We introduce some notations. Let $\lambda$ be a number in $(0,1]$ and let $\mu>0$. Associating with a nonexpansive mapping $S: C \rightarrow H$, we define the mapping $S^{(\lambda, \mu)}: C \rightarrow H$ by $S^{(\lambda, \mu)} \chi:=S x-\lambda \mu F(S x)$ for all $x \in C$, where $F: H \rightarrow H$ is an operator such that, for some positive constants $\kappa, \eta>0, F$ is $k$-Lipschitzian and $\eta$-strongly monotone on $H$, that is, F satisfies the conditions:

$$
\|F x-F y\| \leqslant \kappa\|x-y\| \text { and }\langle F x-F y, x-y\rangle \geqslant \eta\|x-y\|^{2}
$$

for all $x, y \in H$. 
Lemma 2.10 ([21, Lemma 3.1]). $S^{(\lambda, \mu)}$ is a contraction provided $0<\mu<\frac{2 \eta}{\kappa^{2}}$, that is,

$$
\left\|S^{(\lambda, \mu)} x-S^{(\lambda, \mu)} y\right\| \leqslant(1-\lambda \tau)\|x-y\|, \quad \forall x, y \in C,
$$

where $\tau:=1-\sqrt{1-\mu\left(2 \eta-\mu \kappa^{2}\right)} \in(0,1]$.

Remark 2.11. In Lemma 2.10, put $\mathrm{F}=\frac{1}{2} \mathrm{I}$ and $\mu=2$. Then we know that $\mathrm{\kappa}=\eta=\frac{1}{2}, 0<\mu=2<\frac{2 \eta}{\kappa^{2}}=4$, and

$$
\tau=1-\sqrt{1-\mu\left(2 \eta-\mu \kappa^{2}\right)}=1-\sqrt{1-2\left(2 \times \frac{1}{2}-2 \times\left(\frac{1}{2}\right)^{2}\right)}=1 .
$$

Lemma 2.12 ([20]). Let $\left\{a_{k}\right\}$ be a sequence of nonnegative real numbers satisfying the property

$$
a_{k+1} \leqslant\left(1-s_{k}\right) a_{k}+s_{k} t_{k}+\delta_{k}, \quad \forall k \geqslant 0,
$$

where $\left\{s_{k}\right\},\left\{t_{k}\right\}$, and $\left\{\delta_{k}\right\}$ are sequences of real numbers such that

(i) $\left\{s_{k}\right\} \subset[0,1]$ and $\sum_{k=0}^{\infty} s_{k}=\infty$;

(ii) either $\lim \sup _{k \rightarrow \infty} t_{k} \leqslant 0$, or $\sum_{k=0}^{\infty}\left|s_{k} t_{k}\right|<\infty$;

(iii) $\sum_{k=0}^{\infty} \delta_{k}<\infty$ with $\delta_{k} \geqslant 0, \forall k \geqslant 0$.

Then, $\lim _{k \rightarrow \infty} a_{k}=0$.

Lemma 2.13 ([8]). Let $\mathrm{H}$ be a real Hilbert space. Then the following hold:

(a) $\|x-y\|^{2}=\|x\|^{2}-\|y\|^{2}-2\langle x-y, y\rangle$ for all $x, y \in H$;

(b) $\|\lambda x+\mu y\|^{2}=\lambda\|x\|^{2}+\mu\|y\|^{2}-\lambda \mu\|x-y\|^{2}$ for all $x, y \in H$ and $\lambda, \mu \in[0,1]$ with $\lambda+\mu=1$;

(c) if $\left\{x_{k}\right\}$ is a sequence in $\mathrm{H}$ such that $\mathrm{x}_{\mathrm{k}} \rightarrow \mathrm{x}$, it follows that

$$
\limsup _{k \rightarrow \infty}\left\|x_{k}-y\right\|^{2}=\limsup _{k \rightarrow \infty}\left\|x_{k}-x\right\|^{2}+\|x-y\|^{2}, \quad \forall y \in H .
$$

\section{Iterative algorithm and convergence criteria}

Let $\mathrm{H}$ be a real Hilbert space. In this section, we always assume the following.

$\mathrm{T}_{i}: \mathrm{H} \rightarrow \mathrm{H}$ is a nonexpansive mapping for each $i=1, \ldots, N$ such that $\cap_{i=1}^{N} \operatorname{Fix}\left(\mathrm{T}_{i}\right) \neq \emptyset$;

$\mathcal{A}: \mathrm{H} \rightarrow \mathrm{H}$ is $\bar{\eta}$-strongly monotone and $\mathrm{L}_{1}$-Lipschitz continuous, and $\mathrm{F}: \mathrm{H} \rightarrow \mathrm{H}$ is $\eta$-strongly monotone and $\mathrm{k}$-Lipschitzian;

$\left\{\alpha_{\mathrm{k}}\right\},\left\{\beta_{\mathrm{k}}\right\} \subset(0,1]$ and constants $\mu, \nu>0$ satisfy the conditions (A1)-(A3):

(A1) $\mu \in\left(0,2 \eta / \kappa^{2}\right)$ and $v \in\left(0,2 \bar{\eta} / L_{1}^{2}\right)$,

(A2) $\lim _{k \rightarrow \infty}\left(\alpha_{k}-\alpha_{k+N}\right) / \alpha_{k+N}=0$ or $\sum_{k=0}^{\infty}\left|\alpha_{k}-\alpha_{k+N}\right|<\infty$,

(A3) $\lim _{k \rightarrow \infty}\left(\beta_{k}-\beta_{k+N}\right) / \alpha_{k+N}=0$ or $\sum_{k=0}^{\infty}\left|\beta_{k+N}-\beta_{k}\right|<\infty$;

$A: H \rightarrow H$ and $B: H \rightarrow H$ are two mappings such that the hypotheses (H1)-(H4) hold:

(H1) $B$ is monotone on $H$,

(H2) $A$ is $\beta$-inverse-strongly monotone on $\mathrm{H}$,

(H3) B is $\mathrm{L}_{2}$-Lipschitz continuous on $\mathrm{H}$,

(H4) $\Omega:=\operatorname{VI}\left(\mathrm{VI}\left(\cap_{i=1}^{\mathrm{N}} \operatorname{Fix}\left(\mathrm{T}_{i}\right), \mathrm{B}\right), \mathrm{A}\right) \neq \emptyset$.

Next, we introduce the following triple hierarchical variational inequality problem (THVIP) defined over the common fixed point set of finitely many nonexpansive mappings.

Problem 3.1. The objective is to find

$$
x^{*} \in \Omega=\operatorname{VI}\left(\operatorname{VI}\left(\cap_{i=1}^{N} \operatorname{Fix}\left(T_{i}\right), B\right), A\right)=\left\{x^{*} \in \operatorname{VI}\left(\cap_{i=1}^{N} \operatorname{Fix}\left(T_{i}\right), B\right):\left\langle A x^{*}, x-x^{*}\right\rangle \geqslant 0, \forall x \in \operatorname{VI}\left(\cap_{i=1}^{N} \operatorname{Fix}\left(T_{i}\right), B\right)\right\} .
$$

That is, the $\Omega$ is the solution set of the THVIP: find $x^{*} \in \operatorname{VI}\left(\cap_{i=1}^{N} \operatorname{Fix}\left(T_{i}\right), B\right)$ such that

$$
\left\langle A x^{*}, x-x^{*}\right\rangle \geqslant 0, \quad \forall x \in \operatorname{VI}\left(\cap_{i=1}^{N} \operatorname{Fix}\left(T_{i}\right), B\right),
$$

where $\operatorname{VI}\left(\cap_{i=1}^{N} \operatorname{Fix}\left(T_{i}\right), B\right)$ denotes the set of solutions of the VIP: find $y^{*} \in \cap_{i=1}^{N} \operatorname{Fix}\left(T_{i}\right)$ such that 


$$
\left\langle B y^{*}, y-y^{*}\right\rangle \geqslant 0, \quad \forall y \in \cap_{i=1}^{N} \operatorname{Fix}\left(T_{i}\right) .
$$

It is worth pointing out that Problem 3.1 is very different from Problem 1.9 because the solution set of Problem 3.1 may not be a singleton but the solution set of Problem 1.9 must be a singleton.

Algorithm 3.2. Initialization: choose $u \in H, x_{0} \in H, k=0,0<\lambda \leqslant 2 \beta$, positive sequences $\left\{\delta_{k}\right\},\left\{\lambda_{k}\right\},\left\{\alpha_{k}\right\}$, $\left\{\beta_{\mathrm{k}}\right\},\left\{\gamma_{\mathrm{k}}\right\}$, and $\left\{\bar{\epsilon}_{\mathrm{k}}\right\}$ such that

$$
\left\{\begin{array}{l}
\lim _{k \rightarrow \infty} \delta_{k}=0, \quad \sum_{k=0}^{\infty} \bar{\epsilon}_{k}<\infty, \quad \sum_{k=0}^{\infty} \lambda_{k}<\infty \\
\alpha_{k}+\beta_{k}+\gamma_{k}=1, \quad \forall k \geqslant 0, \quad \lim _{k \rightarrow \infty} \beta_{k}=\xi \in\left(0, \frac{1}{2}\right], \\
\sum_{k=0}^{\infty} \alpha_{k}=\infty, \quad \lim _{k \rightarrow \infty} \alpha_{k}=0, \quad \lambda_{k}=0\left(\alpha_{k}\right), \quad \lambda_{k} \leqslant \min \left\{1, \frac{1}{L_{2}}\right\}, \quad \forall k \geqslant 0
\end{array}\right.
$$

Step 1. Compute

$$
\left\{\begin{array}{l}
u_{k}:=\beta_{k} x_{k}+\left(1-\beta_{k}\right)\left(I-\lambda_{k} \mu F\right) T_{[k+1]} u_{k} \\
v_{k}:=u_{k}-\alpha_{k} v \mathcal{A} u_{k} \\
y_{k}:=P_{\cap_{i=1}^{N} \operatorname{Fix}\left(T_{i}\right)}\left(v_{k}-\lambda_{k} B v_{k}\right) \\
z_{k}:=P_{\cap_{i=1}^{N} \operatorname{Fix}\left(T_{i}\right)}\left(v_{k}-\lambda_{k} B y_{k}\right)
\end{array}\right.
$$

Step 2. Inner loop $j=0,1, \ldots$. Compute

$$
\left\{\begin{array}{l}
x_{k, 0}:=z_{k}-\lambda A z_{k}, \\
y_{k, j}:=P_{\cap i=1}^{N} \operatorname{Fix}\left(T_{i}\right) \\
\left.x_{k, j+1}:=\alpha_{j, j} x_{k, 0}+\beta_{j} x_{k, j} B x_{k, j}\right), \\
\text { If } \quad\left\|x_{k, j+1}-P_{\cap^{\prime}\left(\cap_{i=1}^{N} \operatorname{Fix}\left(T_{i}\right), B\right.} x_{k, 0}\right\| \leqslant \bar{\epsilon}_{k} \quad \text { then set } h_{k}:=x_{k, j+1} \text { and go to Step } 3 . \\
\text { Otherwise, increase } j \text { by } 1 \text { and repeat the inner loop Step } 2 .
\end{array}\right.
$$

Step 3. Set $x_{k+1}:=\alpha_{k} u+\beta_{k} x_{k}+\gamma_{k} h_{k}$. Then increase $k$ by 1 and go to Step 1 .

We observe that by Lemma 2.10 for every $z \in H$ and $t \in(0,1]$, the mapping $S_{t}: H \rightarrow H$ defined by $S_{t} x:=t z+(1-t) S^{(\lambda, \mu)} x$ satisfies

$$
\left\|S_{t} x-S_{t} y\right\|=(1-t)\left\|S^{(\lambda, \mu)} x-S^{(\lambda, \mu)} y\right\| \leqslant(1-t)(1-\lambda \tau)\|x-y\|, \quad \forall x, y \in H,
$$

where $0<\lambda \leqslant 1,0<\mu<2 \eta / \kappa^{2}$, and $\tau=1-\sqrt{1-\mu\left(2 \eta-\mu \kappa^{2}\right)} \in(0,1]$. By the Banach Contraction Principle, there exists a unique $x_{t} \in H$ satisfying the equation

$$
x_{t}=t u+(1-t) S^{(\lambda, \mu)} x_{t}
$$

This shows that the implicit iteration step with perturbed mapping F in Step 1 of Algorithm 3.2 is welldefined. Thus, Algorithm 3.2 can be employed for the approximation of solutions of Problem 3.1.

Let $\mathrm{C}$ be a nonempty closed convex subset of $\mathrm{H}, \mathrm{B}: \mathrm{C} \rightarrow \mathrm{H}$ be monotone and $\mathrm{L}_{2}$-Lipschitz continuous on $C$, and $S: C \rightarrow C$ be a nonexpansive mapping such that $\operatorname{VI}(C, B) \cap \operatorname{Fix}(S) \neq \emptyset$. Let the sequences $\left\{x_{n}\right\}$ and $\left\{y_{n}\right\}$ be generated by

$$
\left\{\begin{array}{l}
x_{0} \in C \quad \text { chosen arbitrarily, } \\
y_{k}=P_{C}\left(x_{k}-\delta_{k} B x_{k}\right), \\
x_{k+1}=\alpha_{k} x_{0}+\beta_{k} x_{k}+\gamma_{k} S_{C}\left(x_{k}-\delta_{k} B y_{k}\right), \quad \forall k \geqslant 0
\end{array}\right.
$$


where $\left\{\alpha_{\mathrm{k}}\right\},\left\{\beta_{\mathrm{k}}\right\},\left\{\gamma_{\mathrm{k}}\right\}$, and $\left\{\delta_{\mathrm{k}}\right\}$ satisfy the following conditions:

$$
\left\{\begin{array}{l}
\delta_{k}>0, \quad \forall k \geqslant 0, \quad \lim _{k \rightarrow \infty} \delta_{k}=0, \\
\alpha_{k}+\beta_{k}+\gamma_{k}=1, \quad \forall k \geqslant 0, \\
\sum_{k=0}^{\infty} \alpha_{k}=\infty, \quad \lim _{k \rightarrow \infty} \alpha_{k}=0, \\
0<\liminf _{k \rightarrow \infty} \leqslant \limsup _{k \rightarrow \infty} \beta_{k}<1 .
\end{array}\right.
$$

Under these conditions, Yao et al. [25] proved that the sequences $\left\{x_{k}\right\}$ and $\left\{y_{k}\right\}$ converge strongly to the

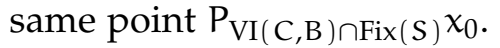

Applying these iteration sequences with $S$ being the identity mapping, we have the following lemma.

Lemma 3.3. Suppose that the hypotheses (H1)-(H4) hold. Then the sequence $\left\{x_{k, j}\right\}$ generated by Algorithm 3.2 converges strongly to the point $\mathrm{P}_{\mathrm{VI}\left(\cap_{i=1}^{N} \operatorname{Fix}\left(\mathrm{T}_{i}\right), \mathrm{B}\right)}\left(z_{\mathrm{k}}-\lambda \mathrm{A} z_{\mathrm{k}}\right)$ as $\mathrm{j} \rightarrow \infty$. Consequently, we have

$$
\left\|h_{k}-P_{V I\left(\cap \cap_{i=1}^{N} \operatorname{Fix}\left(T_{i}\right), B\right)}\left(z_{k}-\lambda A z_{k}\right)\right\| \leqslant \bar{\epsilon}_{k}, \quad \forall k \geqslant 0 .
$$

In the sequel we always suppose that the inner loop in Algorithm 3.2 terminates after a finite number of steps. This assumption, by Lemma 3.3, is satisfied when $B$ is monotone on $\cap_{i=1}^{N} \operatorname{Fix}\left(T_{i}\right)$.

Lemma 3.4. Let the sequences $\left\{v_{\mathrm{k}}\right\},\left\{\mathrm{y}_{\mathrm{k}}\right\}$, and $\left\{z_{\mathrm{k}}\right\}$ be generated by Algorithm 3.2, B be $\mathrm{L}_{2}$-Lipschitzian and monotone on $\mathrm{H}$, and $\mathrm{p} \in \mathrm{VI}\left(\cap_{i=1}^{\mathrm{N}} \operatorname{Fix}\left(\mathrm{T}_{i}\right), \mathrm{B}\right)$. Then, we have

$$
\left\|z_{k}-p\right\|^{2} \leqslant\left\|v_{k}-p\right\|^{2}-\left(1-\lambda_{k} L_{2}\right)\left\|v_{k}-y_{k}\right\|^{2}-\left(1-\lambda_{k} L_{2}\right)\left\|y_{k}-z_{k}\right\|^{2} .
$$

Proof. Let $p \in \operatorname{VI}\left(\cap_{i=1}^{N} \operatorname{Fix}\left(T_{i}\right), B\right)$. That means $\langle B p, x-p\rangle \geqslant 0$ for all $x \in \cap_{i=1}^{N} \operatorname{Fix}\left(T_{i}\right)$. Then, for each $\lambda_{k}>0$, $p$ satisfies the fixed point equation $p=P_{\cap_{i=1}^{N} \operatorname{Fix}\left(T_{i}\right)}\left(p-\lambda_{k} B p\right)$. Since $B$ is monotone on $H$ and $p \in \operatorname{VI}\left(\cap_{i=1}^{N} \operatorname{Fix}\left(T_{i}\right), B\right)$, we have $\left\langle B y_{k}, y_{k}-p\right\rangle \geqslant\left\langle B p, y_{k}-p\right\rangle \geqslant 0$. Then, applying Proposition 2.1 (ii) with $v_{k}-\lambda_{k} B y_{k}$ and $p$, we obtain

$$
\begin{aligned}
\left\|z_{k}-p\right\|^{2} \leqslant & \left\|v_{k}-\lambda_{k} B y_{k}-p\right\|^{2}-\left\|v_{k}-\lambda_{k} B y_{k}-z_{k}\right\|^{2} \\
= & \left\|v_{k}-p\right\|^{2}-2 \lambda_{k}\left\langle B y_{k}, v_{k}-p\right\rangle+\lambda_{k}^{2}\left\|B y_{k}\right\|^{2}-\left\|v_{k}-z_{k}\right\|^{2}-\lambda_{k}^{2}\left\|B y_{k}\right\|^{2} \\
& +2 \lambda_{k}\left\langle B y_{k}, v_{k}-z_{k}\right\rangle \\
= & \left\|v_{k}-p\right\|^{2}-\left\|v_{k}-z_{k}\right\|^{2}+2 \lambda_{k}\left\langle B y_{k}, p-z_{k}\right\rangle \\
= & \left\|v_{k}-p\right\|^{2}-\left\|v_{k}-z_{k}\right\|^{2}+2 \lambda_{k}\left\langle B y_{k}, p-y_{k}\right\rangle+2 \lambda_{k}\left\langle B y_{k}, y_{k}-z_{k}\right\rangle \\
\leqslant & \left\|v_{k}-p\right\|^{2}-\left\|v_{k}-z_{k}\right\|^{2}+2 \lambda_{k}\left\langle B y_{k}, y_{k}-z_{k}\right\rangle .
\end{aligned}
$$

Applying Proposition 2.1, we also have $\left\langle v_{k}-\lambda_{k} B v_{k}-y_{k}, z_{k}-y_{k}\right\rangle \leqslant 0$. Combining this inequality with (3.2) and observing that $B$ is $L_{2}$-Lipschitz continuous on $H$, we obtain

$$
\begin{aligned}
\left\|z_{k}-p\right\|^{2} \leqslant & \left\|v_{k}-p\right\|^{2}-\left\|\left(v_{k}-y_{k}\right)+\left(y_{k}-z_{k}\right)\right\|^{2}+2 \lambda_{k}\left\langle B y_{k}, y_{k}-z_{k}\right\rangle \\
= & \left\|v_{k}-p\right\|^{2}-\left\|v_{k}-y_{k}\right\|^{2}-\left\|y_{k}-z_{k}\right\|^{2}-2\left\langle v_{k}-y_{k}, y_{k}-z_{k}\right\rangle+2 \lambda_{k}\left\langle B y_{k}, y_{k}-z_{k}\right\rangle \\
= & \left\|v_{k}-p\right\|^{2}-\left\|v_{k}-y_{k}\right\|^{2}-\left\|y_{k}-z_{k}\right\|^{2}-2\left\langle v_{k}-\lambda_{k} B y_{k}-y_{k}, y_{k}-z_{k}\right\rangle \\
= & \left\|v_{k}-p\right\|^{2}-\left\|v_{k}-y_{k}\right\|^{2}-\left\|y_{k}-z_{k}\right\|^{2}-2\left\langle v_{k}-\lambda_{k} B v_{k}-y_{k}, y_{k}-z_{k}\right\rangle \\
& +2 \lambda_{k}\left\langle B v_{k}-B y_{k}, z_{k}-y_{k}\right\rangle \\
\leqslant & \left\|v_{k}-p\right\|^{2}-\left\|v_{k}-y_{k}\right\|^{2}-\left\|y_{k}-z_{k}\right\|^{2}+2 \lambda_{k}\left\langle B v_{k}-B y_{k}, z_{k}-y_{k}\right\rangle \\
\leqslant & \left\|v_{k}-p\right\|^{2}-\left\|v_{k}-y_{k}\right\|^{2}-\left\|y_{k}-z_{k}\right\|^{2}+2 \lambda_{k}\left\|B v_{k}-B y_{k}\right\|\left\|z_{k}-y_{k}\right\| \\
\leqslant & \left\|v_{k}-p\right\|^{2}-\left\|v_{k}-y_{k}\right\|^{2}-\left\|y_{k}-z_{k}\right\|^{2}+2 \lambda_{k} L_{2}\left\|v_{k}-y_{k}\right\|\left\|z_{k}-y_{k}\right\| \\
\leqslant & \left\|v_{k}-p\right\|^{2}-\left\|v_{k}-y_{k}\right\|^{2}-\left\|y_{k}-z_{k}\right\|^{2}+\lambda_{k} L_{2}\left(\left\|v_{k}-y_{k}\right\|^{2}+\left\|z_{k}-y_{k}\right\|^{2}\right) \\
\leqslant & \left\|v_{k}-p\right\|^{2}-\left(1-\lambda_{k} L_{2}\right)\left\|v_{k}-y_{k}\right\|^{2}-\left(1-\lambda_{k} L_{2}\right)\left\|y_{k}-z_{k}\right\|^{2} .
\end{aligned}
$$


Lemma 3.5. Suppose that the conditions (A1) and (H1)-(H4) hold. Then the sequence $\left\{x_{\mathrm{k}}\right\}$ generated by Algorithm 3.2 is bounded.

Proof. Since $\lim _{k \rightarrow \infty} \beta_{k}=\xi \in\left(0, \frac{1}{2}\right]$, we may assume, without loss of generality, that $\left\{\beta_{k}\right\} \subset[a, b] \subset(0,1)$. Take an arbitrary $p \in \Omega:=\operatorname{VI}\left(\operatorname{VI}\left(\cap_{i=1}^{N} \operatorname{Fix}\left(T_{i}\right), B\right), A\right)$. We put $S^{\left(\alpha_{k}, v\right)} \chi:=\left(I-\alpha_{k} v \mathcal{A}\right) \chi$ for all $x \in H$. Then $S^{\left(\alpha_{k}, v\right)} u_{k}=\left(I-\alpha_{k} v \mathcal{A}\right) u_{k}$ and $S^{\left(\alpha_{k}, v\right)} p=\left(I-\alpha_{k} v \mathcal{A}\right) p$. Utilizing Lemma 2.10 and the $\bar{\eta}$-strong monotonicity and $\mathrm{L}_{1}$-Lipschitz continuity of $\mathcal{A}$ we have

$$
\begin{aligned}
\left\|v_{k}-p\right\| & \leqslant\left\|S^{\left(\alpha_{k}, v\right)} u_{k}-S^{\left(\alpha_{k}, v\right)} p\right\|+\left\|S^{\left(\alpha_{k}, v\right)} p-p\right\| \\
& \leqslant\left(1-\alpha_{k} \sigma\right)\left\|u_{k}-p\right\|+\alpha_{k} v\|\mathcal{A} p\| \leqslant \max \left\{\left\|u_{k}-p\right\|, \frac{v\|\mathcal{A} p\|_{0}}{\sigma}\right\}
\end{aligned}
$$

where $\sigma:=1-\sqrt{1-v\left(2 \bar{\eta}-v L_{1}^{2}\right)}$.

Also, we put $T^{\left(\lambda_{k}, \mu\right)} x:=\left(I-\lambda_{k} \mu F\right) T_{[k+1]} x$ for all $x \in H$. Then $T^{\left(\lambda_{k}, \mu\right)} u_{k}=\left(I-\lambda_{k} \mu F\right) T_{[k+1]} u_{k}$ and $\mathrm{T}^{\left(\lambda_{k}, \mu\right)} p=\left(\mathrm{I}-\lambda_{k} \mu \mathrm{F}\right) p$ for all $k \geqslant 0$. Since $p \in \Omega:=\operatorname{VI}\left(\operatorname{VI}\left(\cap_{i=1}^{N} \operatorname{Fix}\left(T_{i}\right), B\right), A\right)$, we obtain that $T_{[k+1]} p=p$ and

$$
\left\|\mathfrak{u}_{k}-p\right\| \leqslant \beta_{k}\left\|x_{k}-p\right\|+\left(1-\beta_{k}\right)\left\|T^{\left(\lambda_{k}, \mu\right)} \mathfrak{u}_{k}-p\right\| .
$$

Utilizing Lemma 2.10 we have

$$
\left\|T^{\left(\lambda_{k}, \mu\right)} u_{k}-p\right\| \leqslant\left\|T^{\left(\lambda_{k}, \mu\right)} u_{k}-T^{\left(\lambda_{k}, \mu\right)} p\right\|+\left\|T^{\left(\lambda_{k}, \mu\right)} p-p\right\| \leqslant\left(1-\lambda_{k} \tau\right)\left\|u_{k}-p\right\|+\lambda_{k} \mu\|F p\|,
$$

where $\tau=1-\sqrt{1-\mu\left(2 \eta-\mu \kappa^{2}\right)}$. This together with (3.5) yields

$$
\begin{aligned}
\left\|u_{k}-p\right\| & \leqslant \beta_{k}\left\|x_{k}-p\right\|+\left(1-\beta_{k}\right)\left[\left(1-\lambda_{k} \tau\right)\left\|u_{k}-p\right\|+\lambda_{k} \mu\|F p\|\right], \\
& \leqslant \beta_{k}\left\|x_{k}-p\right\|+\left(1-\beta_{k}\right)\left\|u_{k}-p\right\|+\left(1-\beta_{k}\right) \lambda_{k} \mu\|F p\|,
\end{aligned}
$$

and so

$$
\left\|u_{k}-p\right\| \leqslant\left\|x_{k}-p\right\|+\left(1-\beta_{k}\right) \lambda_{k} \frac{\mu\|F p\|}{\beta_{k}} \leqslant\left\|x_{k}-p\right\|+\lambda_{k} \frac{\mu\|F p\|}{a} .
$$

Combining (3.4) and (3.6), we get

$$
\left\|v_{k}-p\right\| \leqslant \max \left\{\left\|x_{k}-p\right\|, \frac{v\|\mathcal{A} p\|}{\sigma}\right\}+\lambda_{k} \frac{\mu\|F p\|}{a} .
$$

Since $p \in \operatorname{VI}\left(\operatorname{VI}\left(B, \cap \cap_{i=1}^{N} \operatorname{Fix}\left(T_{i}\right)\right), A\right)$, we have $\langle A p, x-p\rangle \geqslant 0$ for all $x \in \operatorname{VI}\left(\cap_{i=1}^{N} \operatorname{Fix}\left(T_{i}\right)\right.$, B) which implies $p=P_{\mathrm{VI}\left(\cap_{i=1}^{N} \operatorname{Fix}\left(T_{i}\right), B\right)}(p-\lambda A p)$. Then, from (2.1), Proposition 2.1 (iii), $\beta$-inverse strong monotonicity of $A$, and $0<\lambda \leqslant 2 \beta$, it follows that

$$
\begin{aligned}
\left\|P_{\mathrm{VI}\left(\cap_{i=1}^{N} \operatorname{Fix}\left(T_{i}\right), \mathrm{B}\right)}\left(z_{k}-\lambda A z_{k}\right)-p\right\|^{2} & =\left\|P_{\mathrm{VI}\left(\cap_{i=1}^{N} \operatorname{Fix}\left(T_{i}\right), \mathrm{B}\right)}\left(z_{k}-\lambda A z_{k}\right)-P_{\mathrm{VI}\left(\cap_{i=1}^{N} \operatorname{Fix}\left(T_{i}\right), B\right)}(p-\lambda A p)\right\|^{2} \\
& \leqslant\left\|(I-\lambda A) z_{k}-(I-\lambda A) p\right\|^{2} \\
& \leqslant\left\|z_{k}-p\right\|^{2}+\lambda(\lambda-2 \beta)\left\|A z_{k}-A p\right\|^{2} \\
& \leqslant\left\|z_{k}-p\right\|^{2} .
\end{aligned}
$$

Utilizing (3.3), (3.7), (3.8), and the assumptions $0<\lambda \leqslant 2 \beta, \sum_{k=0}^{\infty} \lambda_{k}<\infty, \sum_{k=0}^{\infty} \bar{\epsilon}_{k}<\infty$, we obtain that

$$
\begin{aligned}
\left\|x_{k+1}-p\right\|= & \left\|\alpha_{k} u+\beta_{k} x_{k}+\gamma_{k} h_{k}-p\right\| \\
\leqslant & \left.\alpha_{k}\|u-p\|+\beta_{k}\left\|x_{k}-p\right\|+\gamma_{k} \| h_{k}-P_{V I(\cap i=1}^{N} \operatorname{Fix}\left(T_{i}\right), B\right) \\
& +\gamma_{k}\left\|P_{V I}\left(\cap_{k}-\lambda A z_{k}\right)\right\| \\
\leqslant & \alpha_{k}\|u-p\|+\beta_{k}\left\|x_{k}-p\right\|+\gamma_{k} \bar{\epsilon}_{k}+\gamma_{k}\left\|v_{k}-p\right\|
\end{aligned}
$$




$$
\begin{aligned}
& \leqslant \alpha_{k}\|u-p\|+\left(\beta_{k}+\gamma_{k}\right) \max \left\{\left\|x_{k}-p\right\|, \frac{v\|\mathcal{A} p\|}{\sigma}\right\}+\bar{\epsilon}_{k}+\lambda_{k} \frac{\mu\|F p\|}{a} \\
& =\alpha_{k}\|u-p\|+\left(1-\alpha_{k}\right) \max \left\{\left\|x_{k}-p\right\|, \frac{v\|\mathcal{A} p\|}{\sigma}\right\}+\bar{\epsilon}_{k}+\lambda_{k} \frac{\mu\|F p\|}{a} \\
& \leqslant \max \left\{\left\|x_{k}-p\right\|,\|u-p\|, \frac{v\|\mathcal{A} p\|}{\sigma}\right\}+\bar{\epsilon}_{k}+\lambda_{k} \frac{\mu\|F p\|}{a} \\
& \leqslant \max \left\{\left\|x_{0}-p\right\|,\|u-p\|, \frac{v\|\mathcal{A} p\|}{\sigma}\right\}+\sum_{k=0}^{\infty}\left(\bar{\epsilon}_{k}+\lambda_{k} \frac{\mu\|F p\|}{a}\right)
\end{aligned}
$$

which shows that the sequence $\left\{x_{k}\right\}$ is bounded, and so are the sequences $\left\{u_{k}\right\},\left\{v_{k}\right\},\left\{y_{k}\right\}$, and $\left\{z_{k}\right\}$.

Lemma 3.6. Suppose that the conditions (H1)-(H4) hold. Assume that the sequences $\left\{v_{\mathrm{k}}\right\}$ and $\left\{z_{\mathrm{k}}\right\}$ are generated by Algorithm 3.2. Then, we have

$$
\left\{\begin{array}{l}
\left\|z_{k+N}-z_{k}\right\| \leqslant\left(1+\lambda_{k+N} L_{2}\right)\left\|v_{k+N}-v_{k}\right\|+\lambda_{k}\left\|B y_{k}\right\|+\lambda_{k+N}\left(\left\|B v_{k+N}\right\|+\left\|B y_{k+N}\right\|+\left\|B v_{k}\right\|\right), \\
\left\|z_{k+1}-z_{k}\right\| \leqslant\left(1+\lambda_{k+1} L_{2}\right)\left\|v_{k+1}-v_{k}\right\|+\lambda_{k}\left\|B y_{k}\right\|+\lambda_{k+1}\left(\left\|B v_{k+1}\right\|+\left\|B y_{k+1}\right\|+\left\|B v_{k}\right\|\right) .
\end{array}\right.
$$

Proof. Since $\lim _{k \rightarrow \infty} \beta_{k}=\xi \in\left(0, \frac{1}{2}\right]$, we may assume, without loss of generality, that $\left\{\beta_{k}\right\} \subset[a, b] \subset(0,1)$ for all $k \geqslant 0$. Taking into account the $L_{2}$-Lipschitzian property of $B$, for each $x, y \in H$ we have

$$
\left\|\left(I-\lambda_{k} B\right) x-\left(I-\lambda_{k} B\right) y\right\|=\left\|x-y-\lambda_{k}(B x-B y)\right\| \leqslant\left(1+\lambda_{k} L_{2}\right)\|x-y\| .
$$

Combining this inequality with Proposition 2.1 (iii), we have

$$
\begin{aligned}
\left\|z_{k+N}-z_{k}\right\| & =\left\|P_{\cap_{i=1}^{N} \operatorname{Fix}\left(T_{i}\right)}\left(v_{k+N}-\lambda_{k+N} B y_{k+N}\right)-P_{\cap_{i=1}^{N} \operatorname{Fix}\left(T_{i}\right)}\left(v_{k}-\lambda_{k} B y_{k}\right)\right\| \\
& \leqslant\left\|\left(v_{k+N}-\lambda_{k+N} B y_{k+N}\right)-v_{k}+\lambda_{k} B y_{k}\right\| \\
& =\left\|\left(v_{k+N}-\lambda_{k+N} B v_{k+N}\right)-\left(v_{k}-\lambda_{k+N} B v_{k}\right)+\lambda_{k+N}\left(B v_{k+N}-B y_{k+N}-B v_{k}\right)+\lambda_{k} B y_{k}\right\| \\
& \leqslant\left(1+\lambda_{k+N} L_{2}\right)\left\|v_{k+N}-v_{k}\right\|+\lambda_{k}\left\|B y_{k}\right\|+\lambda_{k+N}\left(\left\|B v_{k+N}\right\|+\left\|B y_{k+N}\right\|+\left\|B v_{k}\right\|\right) .
\end{aligned}
$$

Similarly, we can derive

$$
\left\|z_{k+1}-z_{k}\right\| \leqslant\left(1+\lambda_{k+1} \mathrm{~L}_{2}\right)\left\|v_{k+1}-v_{k}\right\|+\lambda_{k}\left\|B y_{k}\right\|+\lambda_{k+1}\left(\left\|B v_{k+1}\right\|+\left\|B y_{k+1}\right\|+\left\|B v_{k}\right\|\right) .
$$

This completes the proof.

Proposition 3.7 ([18]). Let $\left\{x_{k}\right\}$ and $\left\{y_{k}\right\}$ be two bounded sequences in a real Banach space $X$. Let $\left\{\beta_{k}\right\}$ be a sequence in $[0,1]$. Suppose that $0<\liminf _{k \rightarrow \infty} \beta_{k} \leqslant \limsup _{k \rightarrow \infty} \beta_{k}<1, x_{k+1}=\left(1-\beta_{k}\right) y_{k}+\beta_{k} x_{k}$ and $\lim \sup _{k \rightarrow \infty}\left(\left\|y_{k+1}-y_{k}\right\|-\left\|x_{k+1}-x_{k}\right\|\right) \leqslant 0$. Then, $\lim _{k \rightarrow \infty}\left\|y_{k}-x_{k}\right\|=0$.

Lemma 3.8. Suppose that the conditions (H1)-(H4) hold. Assume that the sequence $\left\{x_{\mathrm{k}}\right\}$ is generated by Algorithm 3.2. Then,

(i) if the conditions (A1)-(A3) hold, then $\lim _{k \rightarrow \infty}\left\|x_{k+N}-x_{k}\right\|=0$;

(ii) if the condition (A1) holds and $\lim _{k \rightarrow \infty}\left\|x_{k}-v_{k}\right\|=0$, then $\lim _{k \rightarrow \infty}\left\|x_{k+1}-x_{k}\right\|=0$.

Proof.

(i) Assume that the conditions (A1)-(A3) hold. We write $x_{k+1}=\left(1-\beta_{k}\right) w_{k}+\beta_{k} x_{k}$ for all $k \geqslant 0$. Then, we have

$$
\begin{aligned}
w_{k+N}-w_{k} & =\frac{\alpha_{k+N} u+\gamma_{k+N} h_{k+N}}{1-\beta_{k+N}}-\frac{\alpha_{k} u+\gamma_{k} h_{k}}{1-\beta_{k}} \\
& =\left(\frac{\alpha_{k+N}}{1-\beta_{k+N}}-\frac{\alpha_{k}}{1-\beta_{k}}\right) u+\left(\frac{\gamma_{k+N}}{1-\beta_{k+N}}-\frac{\gamma_{k}}{1-\beta_{k}}\right) h_{k}+\frac{\gamma_{k+N}}{1-\beta_{k+N}}\left(h_{k+N}-h_{k}\right) .
\end{aligned}
$$


Note that, for $0<\lambda \leqslant 2 \beta$, we have from (2.1) that

$$
\left\|\mathrm{P}_{\mathrm{VI}\left(\cap_{i=1}^{\mathrm{N}} \operatorname{Fix}\left(\mathrm{T}_{\mathrm{i}}\right), \mathrm{B}\right)}\left(z_{\mathrm{k}+\mathrm{N}}-\lambda A z_{\mathrm{k}+\mathrm{N}}\right)-\mathrm{P}_{\mathrm{VI}\left(\cap_{i=1}^{\mathrm{N}} \operatorname{Fix}\left(\mathrm{T}_{i}\right), \mathrm{B}\right)}\left(z_{k}-\lambda A z_{k}\right)\right\| \leqslant\left\|z_{k+N}-z_{k}\right\| .
$$

Then, utilizing (3.9) and (3.10) we get

$$
\begin{aligned}
\left\|w_{k+N}-w_{k}\right\| & \leqslant \frac{\alpha_{k+N}}{1-\beta_{k+N}}-\frac{\alpha_{k}}{1-\beta_{k}}|\|u\|+| \frac{\gamma_{k+N}}{1-\beta_{k+N}}-\frac{\gamma_{k}}{1-\beta_{k}} \mid\left(\left\|P_{\mathrm{VI}\left(\cap_{i=1}^{N} \operatorname{Fix}\left(T_{i}\right), \mathrm{B}\right)}\left(z_{k}-\lambda A z_{k}\right)\right\|+\bar{\epsilon}_{k}\right) \\
& +\frac{\gamma_{k+N}}{1-\beta_{k+N}}\left\|z_{k+N}-z_{k}\right\|+\frac{\gamma_{k+N}}{1-\beta_{k+N}}\left(\bar{\epsilon}_{k+N}+\bar{\epsilon}_{k}\right) \\
\leqslant & \left|\frac{\alpha_{k+N}}{1-\beta_{k+N}}-\frac{\alpha_{k}}{1-\beta_{k}}\right|\|u\|+\left|\frac{\gamma_{k+N}}{1-\beta_{k+N}}-\frac{\gamma_{k}}{1-\beta_{k}}\right|\left(\left\|P_{V I\left(\cap \cap_{i=1}^{N} \operatorname{Fix}\left(T_{i}\right), B\right)}\left(z_{k}-\lambda A z_{k}\right)\right\|+\bar{\epsilon}_{k}\right) \\
& +\frac{\gamma_{k+N}\left(1+\lambda_{k+N} L_{2}\right)}{1-\beta_{k+N}}\left\|v_{k+N}-v_{k}\right\|+\frac{\gamma_{k+N}}{1-\beta_{k+N}}\left(\bar{\epsilon}_{k+N}+\bar{\epsilon}_{k}\right) \\
& +\frac{\gamma_{k+N}}{1-\beta_{k+N}}\left(\lambda_{k+N}\left(\left\|B v_{k+N}\right\|+\left\|B y_{k+N}\right\|+\left\|B v_{k}\right\|\right)+\lambda_{k}\left\|B y_{k}\right\|\right) \\
= & \left|\frac{\alpha_{k+N}}{1-\beta_{k+N}}-\frac{\alpha_{k}}{1-\beta_{k}}\right|\|u\|+\left|\frac{\alpha_{k+N}}{1-\beta_{k+N}}-\frac{\alpha_{k}}{1-\beta_{k}}\right|\left(\left\|P_{V I\left(\cap \cap_{i=1}^{N} \operatorname{Fix}\left(T_{i}\right), B\right)}\left(z_{k}-\lambda A z_{k}\right)\right\|+\bar{\epsilon}_{k}\right) \\
& +\frac{\gamma_{k+N}\left(1+\lambda_{k+N} L_{2}\right)}{\alpha_{k+N}+\gamma_{k+N}}\left\|v_{k+N}-v_{k}\right\|+\frac{\gamma_{k+N}}{\alpha_{k+N}+\gamma_{k+N}}\left(\bar{\epsilon}_{k+N}+\bar{\epsilon}_{k}\right) \\
& +\frac{\gamma_{k+N}}{\alpha_{k+N}+\gamma_{k+N}}\left(\lambda_{k+N}\left(\left\|B v_{k+N}\right\|+\left\|B y_{k+N}\right\|+\left\|B v_{k}\right\|\right)+\lambda_{k}\left\|B y_{k}\right\|\right) \\
\leqslant & \left(\frac{\left|\alpha_{k+N}-\alpha_{k}\right|}{1-\beta_{k+N}}+\frac{\alpha_{k}\left|\beta_{k+N}-\beta_{k}\right|}{\left(1-\beta_{k+N}\right)\left(1-\beta_{k}\right)}\right)\left(\|u\|+\left\|P_{V I\left(\cap \cap_{i=1}^{N} \operatorname{Fix}\left(T_{i}\right), B\right)}\left(z_{k}-\lambda A z_{k}\right)\right\|+\bar{\epsilon}_{k}\right) \\
& +\left\|v_{k+N}-v_{k}\right\|+\lambda_{k+N} L_{2}\left\|v_{k+N}-v_{k}\right\|+\bar{\epsilon}_{k+N}+\bar{\epsilon}_{k} \\
& +\lambda_{k+N}\left(\left\|B v_{k+N}\right\|+\left\|B y_{k+N}\right\|+\left\|B v_{k}\right\|\right)+\lambda_{k}\left\|B y_{k}\right\| \\
\leqslant & \left\|v_{k+N}-v_{k}\right\|+\left(\left|\alpha_{k+N}-\alpha_{k}\right|+\left|\beta_{k+N}-\beta_{k}\right|\right) \frac{\|u\|+\left\|P_{V I\left(\cap \cap_{i=1}^{N} \operatorname{Fix}\left(T_{i}\right), B\right)}^{1-b}\left(z_{k}-\lambda A z_{k}\right)\right\|+\bar{\epsilon}_{k}}{} \\
& +\bar{\epsilon}_{k+N}+\bar{\epsilon}_{k}+\lambda_{k+N}\left(L_{2}\left\|v_{k+N}-v_{k}\right\|+\left\|B v_{k+N}\right\|+\left\|B y_{k+N}\right\|+\left\|B v_{k}\right\|\right)+\lambda_{k}\left\|B y_{k}\right\| .
\end{aligned}
$$

On the other hand, utilizing Lemma 2.10, from the nonexpansivity of each $T_{i}$ and the $\eta$-strong monotonicity and $k$-Lipschitz continuity of $F$, we conclude that for all $k \geqslant 0$,

$$
\begin{aligned}
\left\|T^{\left(\lambda_{k+N}, \mu\right)} u_{k+N}-T^{\left(\lambda_{k}, \mu\right)} u_{k}\right\|= & \left\|\left(I-\lambda_{k+N} \mu F\right) T_{[k+N+1]} u_{k+N}-\left(I-\lambda_{k} \mu F\right) T_{[k+1]} u_{k}\right\| \\
= & \left\|\left(I-\lambda_{k+N} \mu F\right) T_{[k+1]} u_{k+N}-\left(I-\lambda_{k} \mu F\right) T_{[k+1]} u_{k}\right\| \\
\leqslant & \left\|\left(I-\lambda_{k+N} \mu F\right) T_{[k+1]} u_{k+N}-\left(I-\lambda_{k+N} \mu F\right) T_{[k+1]} u_{k}\right\| \\
& +\left\|\left(I-\lambda_{k+N} \mu F\right) T_{[k+1]} u_{k}-\left(I-\lambda_{k} \mu F\right) T_{[k+1]} u_{k}\right\| \\
\leqslant & \left(1-\lambda_{k+N} \tau\right)\left\|u_{k+N}-u_{k}\right\|+\mid \lambda_{k+N}-\lambda_{k}\left\|\mu F T_{[k+1]} u_{k}\right\|,
\end{aligned}
$$

and hence

$$
\begin{aligned}
\left\|u_{k+N}-u_{k}\right\|= & \left\|\beta_{k+N} x_{k+N}+\left(1-\beta_{k+N}\right) T^{\left(\lambda_{k+N}, \mu\right)} u_{k+N}-\beta_{k} x_{k}-\left(1-\beta_{k}\right) T^{\left(\lambda_{k}, \mu\right)} u_{k}\right\| \\
= & \| \beta_{k}\left(x_{k+N}-x_{k}\right)+\left(1-\beta_{k}\right)\left(T^{\left(\lambda_{k+N}, \mu\right)} u_{k+N}-T^{\left(\lambda_{k}, \mu\right)} u_{k}\right) \\
& +\left(\beta_{k+N}-\beta_{k}\right)\left(x_{k+N}-T^{\left(\lambda_{k+N}, \mu\right)} u_{k+N}\right) \| \\
\leqslant & \beta_{k}\left\|x_{k+N}-x_{k}\right\|+\left(1-\beta_{k}\right)\left\|T^{\left(\lambda_{k+N}, \mu\right)} u_{k+N}-T^{\left(\lambda_{k}, \mu\right)} u_{k}\right\| \\
& +\left|\beta_{k+N}-\beta_{k}\right|\left\|x_{k+N}-T^{\left(\lambda_{k+N}, \mu\right)} u_{k+N}\right\| \\
\leqslant & \beta_{k}\left\|x_{k+N}-x_{k}\right\|+\left(1-\beta_{k}\right)\left[\left(1-\lambda_{k+N} \tau\right)\left\|u_{k+N}-u_{k}\right\|\right. \\
& \left.+\left|\lambda_{k+N}-\lambda_{k}\right|\left\|\mu F T_{[k+1]} u_{k}\right\|\right]+\left|\beta_{k+N}-\beta_{k}\right|\left\|x_{k+N}-T^{\left(\lambda_{k+N}, \mu\right)} u_{k+N}\right\| \\
\leqslant & \beta_{k}\left\|x_{k+N}-x_{k}\right\|+\left(1-\beta_{k}\right)\left\|u_{k+N}-u_{k}\right\|+\left(1-\beta_{k}\right)\left|\lambda_{k+N}-\lambda_{k}\right|\left\|\mu F T_{[k+1]} u_{k}\right\|
\end{aligned}
$$




$$
+\left|\beta_{k+N}-\beta_{k}\right| \mid x_{k+N}-T^{\left(\lambda_{k+N}, \mu\right)} u_{k+N} \|,
$$

which immediately leads to

$$
\begin{aligned}
\left\|u_{k+N}-u_{k}\right\| \leqslant & \left\|x_{k+N}-x_{k}\right\|+\left(1-\beta_{k}\right)\left|\lambda_{k+N}-\lambda_{k}\right| \frac{\left\|\mu F T_{[k+1]} u_{k}\right\|}{\beta_{k}} \\
& +\left|\beta_{k+N}-\beta_{k}\right| \frac{\left\|x_{k+N}-T^{\left(\lambda_{k+N}, \mu\right)} u_{k+N}\right\|}{\beta_{n}} \\
\leqslant & \left\|x_{k+N}-x_{k}\right\|+\left|\lambda_{k+N}-\lambda_{k}\right| \frac{\left\|\mu F T_{[k+1]} u_{k}\right\|}{a} \\
& +\left|\beta_{k+N}-\beta_{k}\right| \frac{\left\|x_{k+N}-\left(I-\lambda_{k+N} \mu F\right) T_{[k+N+1]} u_{k+N}\right\|}{a} \\
\leqslant & \left\|x_{k+N}-x_{k}\right\|+\left|\lambda_{k+N}-\lambda_{k}\right| \frac{\left\|\mu F T_{[k+1]} u_{k}\right\|}{a} \\
& +\left|\beta_{k+N}-\beta_{k}\right| \frac{\left\|x_{k+N}\right\|+\left\|T_{[k+1]} u_{k+N}\right\|+\left\|\mu F T_{[k+1]} u_{k+N}\right\|}{a} .
\end{aligned}
$$

From (3.12) and Lemma 2.10 it is found that

$$
\begin{aligned}
\left\|v_{k+N}-v_{k}\right\|= & \left\|S^{\left(\alpha_{k+N}, \mu_{k+N}\right)} u_{k+N}-S^{\left(\alpha_{k}, \mu_{k}\right)} u_{k}\right\| \\
\leqslant & \left\|S^{\left(\alpha_{k+N}, \mu_{k+N}\right)} u_{k+N}-S^{\left(\alpha_{k+N}, \mu_{k+N}\right)} u_{k}\right\|+\left\|S^{\left(\alpha_{k+N}, \mu_{k+N}\right)} \mathfrak{u}_{k}-S^{\left(\alpha_{k}, \mu_{k}\right)} \mathfrak{u}_{k}\right\| \\
\leqslant & \left(1-\alpha_{k+N} \sigma\right)\left\|\mathfrak{u}_{k+N}-u_{k}\right\|+\left|\alpha_{k+N}-\alpha_{k}\right|\left\|v \mathcal{A} u_{k}\right\| \\
\leqslant & \left(1-\alpha_{k+N} \sigma\right)\left[\left\|x_{k+N}-x_{k}\right\|+\left|\lambda_{k+N}-\lambda_{k}\right| \frac{\left\|\mu F T_{[k+1]} u_{k}\right\|}{a}\right. \\
& \left.+\left|\beta_{k+N}-\beta_{k}\right| \frac{\left\|x_{k+N}\right\|+\left\|T_{[k+1]} u_{k+N}\right\|+\left\|\mu F T_{[k+1]} u_{k+N}\right\|}{a}\right]+\left|\alpha_{k+N}-\alpha_{k}\right|\left\|v \mathcal{A} u_{k}\right\| \\
\leqslant & \left(1-\alpha_{k+N} \sigma\right)\left\|x_{k+N}-x_{k}\right\|+\left|\lambda_{k+N}-\lambda_{k}\right| \frac{\left\|\mu F T_{[k+1]} u_{k}\right\|}{a} \\
& +\left|\beta_{k+N}-\beta_{k}\right| \frac{\left\|x_{k+N}\right\|+\left\|T_{[k+1]} u_{k+N}\right\|+\left\|\mu F T_{[k+1]} u_{k+N}\right\|}{a}+\left|\alpha_{k+N}-\alpha_{k}\right|\left\|v \mathcal{A} u_{k}\right\| \\
\leqslant & \left(1-\alpha_{k+N} \sigma\right)\left\|x_{k+N}-x_{k}\right\|+\left(\lambda_{k+N}+\lambda_{k}+\left|\alpha_{k+N}-\alpha_{k}\right|+\left|\beta_{k+N}-\beta_{k}\right|\right) M_{0},
\end{aligned}
$$

where $\sup _{k \geqslant 0}\left\{\left\|v \mathcal{A} u_{k}\right\|+\frac{\left\|\mu F T_{[k+1]} u_{k}\right\|}{a}+\frac{\left\|x_{k+N}\right\|+\left\|T_{[k+1]} u_{k+N}\right\|+\left\|\mu F T_{[k+1]} u_{k+N}\right\|}{a}\right\} \leqslant M_{0}$ for some $M_{0}>0$. Utilizing the relation $x_{k+1}=\beta_{k} x_{k}+\left(1-\beta_{k}\right) w_{k}$, we obtain from (3.11) and (3.13) that

$$
\begin{aligned}
&\left\|x_{k+N+1}-x_{k+1}\right\| \\
&=\left\|\beta_{k+N} x_{k+N}+\left(1-\beta_{k+N}\right) w_{k+N}-\beta_{k} x_{k}-\left(1-\beta_{k}\right) w_{k}\right\| \\
& \leqslant \beta_{k}\left\|x_{k+N}-x_{k}\right\|+\left(1-\beta_{k}\right)\left\|w_{k+N}-w_{k}\right\|+\left|\beta_{k+N}-\beta_{k}\right|\left\|x_{k+N}-w_{k+N}\right\| \\
& \leqslant \beta_{k}\left\|x_{k+N}-x_{k}\right\|+\left(1-\beta_{k}\right)\left\{\left\|v_{k+N}-v_{k}\right\|+\left(\left|\alpha_{k+N}-\alpha_{k}\right|+\left|\beta_{k+N}-\beta_{k}\right|\right)\right. \\
& \times \frac{1}{1-b}\left(\|u\|+\left\|P_{V I\left(\cap \cap_{i=1}^{N} \operatorname{Fix}\left(T_{i}\right), B\right)}\left(z_{k}-\lambda A z_{k}\right)\right\|+\bar{\epsilon}_{k}\right)+\lambda_{k+N}\left(L_{2}\left\|v_{k+N}-v_{k}\right\|+\left\|B v_{k+N}\right\|\right. \\
&\left.\left.+\left\|B y_{k+N}\right\|+\left\|B v_{k}\right\|\right)+\lambda_{k}\left\|B y_{k}\right\|\right\}+\left|\beta_{k+N}-\beta_{k}\right|\left\|x_{k+N}-w_{k+N}\right\|+\bar{\epsilon}_{k+N}+\bar{\epsilon}_{k} \\
& \leqslant \beta_{k}\left\|x_{k+N}-x_{k}\right\|+\left(1-\beta_{k}\right)\left\{\left(1-\alpha_{k+N} \sigma\right)\left\|x_{k+N}-x_{k}\right\|+\left(\lambda_{k+N}+\lambda_{k}\right.\right. \\
&\left.+\left|\alpha_{k+N}-\alpha_{k}\right|+\left|\beta_{k+N}-\beta_{k}\right|\right) M_{0}+\left(\left|\alpha_{k+N}-\alpha_{k}\right|+\left|\beta_{k+N}-\beta_{k}\right|\right) \\
&+\|u\|+\| P_{V I}\left(\cap_{i=1}^{N} \operatorname{Fix}\left(T_{i}\right), B\right) \\
&\left.\times \frac{1-b}{1}-\lambda A z_{k}\right) \|+\bar{\epsilon}_{k} \\
&\left.\left.+\left\|B y_{k+N}\right\|+\left\|B v_{k}\right\|\right)+\lambda_{k}\left\|B y_{k}\right\|\right\}+\left|\beta_{k+N}-\beta_{k}\right|\left\|x_{k+N}-w_{k+N}\right\|+\bar{\epsilon}_{k+N}\left\|v_{k+N}-v_{k}\right\|+\left\|B v_{k+N}\right\| \\
& \leqslant \beta_{k}\left\|x_{k+N}-x_{k}\right\|+\left(1-\beta_{k}\right)\left(1-\alpha_{k+N} \sigma\right)\left\|x_{k+N}-x_{k}\right\|+\left(\lambda_{k+N}+\lambda_{k}\right. \\
&\left.+\left|\alpha_{k+N}-\alpha_{k}\right|+\left|\beta_{k+N}-\beta_{k}\right|\right) M_{1}+\bar{\epsilon}_{k+N}+\bar{\epsilon}_{k} \\
&=\left(1-\alpha_{k+N}\left(1-\beta_{k}\right) \sigma\right)\left\|x_{k+N}-x_{k}\right\|+\left(\left|\alpha_{k+N}-\alpha_{k}\right|+\left|\beta_{k+N}-\beta_{k}\right|\right) M_{1} \\
&+\left(\lambda_{k+N}+\lambda_{k}\right) M_{1}+\bar{\epsilon}_{k+N}+\bar{\epsilon}_{k}
\end{aligned}
$$


where there is a constant $M_{1}>0$ such that

$$
\begin{aligned}
& \sup _{k \geqslant 0} \mathrm{M}_{0}+\left\|\mathrm{B} y_{k}\right\|+\frac{\|u\|+\left\|\mathrm{P}_{\mathrm{VI}\left(\cap_{i=1}^{N} \operatorname{Fix}\left(\mathrm{T}_{\mathrm{i}}\right), \mathrm{B}\right)}\left(z_{\mathrm{k}}-\lambda \mathrm{A} z_{\mathrm{k}}\right)\right\|+\bar{\epsilon}_{\mathrm{k}}}{1-\mathrm{b}}+\left\|\mathrm{x}_{\mathrm{k}+\mathrm{N}}-w_{\mathrm{k}+\mathrm{N}}\right\| \\
&\left.+\mathrm{L}_{2}\left\|v_{\mathrm{k}+\mathrm{N}}-v_{\mathrm{k}}\right\|+\left\|\mathrm{B} v_{\mathrm{k}+\mathrm{N}}\right\|+\left\|\mathrm{B} y_{k+N}\right\|+\left\|\mathrm{B} v_{\mathrm{k}}\right\|\right\} \leqslant \mathrm{M}_{1} .
\end{aligned}
$$

Applying Lemma 2.12 to (3.14), we deduce from conditions (A2) and (A3) that $\lim _{k \rightarrow \infty}\left\|x_{k+N}-x_{k}\right\|=0$. (ii) Assume that the condition (A1) holds and $\lim _{k \rightarrow \infty}\left\|x_{k}-v_{k}\right\|=0$. Observe that for all $k \geqslant 0$,

$$
\begin{aligned}
w_{k+1}-w_{k} & =\frac{\alpha_{k+1} u+\gamma_{k+1} h_{k+1}}{1-\beta_{k+1}}-\frac{\alpha_{k} u+\gamma_{k} h_{k}}{1-\beta_{k}} \\
& =\left(\frac{\alpha_{k+1}}{1-\beta_{k+1}}-\frac{\alpha_{k}}{1-\beta_{k}}\right) u+\left(\frac{\gamma_{k+1}}{1-\beta_{k+1}}-\frac{\gamma_{k}}{1-\beta_{k}}\right) h_{k}+\frac{\gamma_{k+1}}{1-\beta_{k+1}}\left(h_{k+1}-h_{k}\right) .
\end{aligned}
$$

Utilizing (3.9) and (3.15) we get

$$
\begin{aligned}
\| & w_{k+1}-w_{k}\|-\| x_{k+1}-x_{k} \| \\
\leqslant & \frac{\alpha_{k+1}}{1-\beta_{k+1}}-\frac{\alpha_{k}}{1-\beta_{k}}|\|u\|+| \frac{\gamma_{k+1}}{1-\beta_{k+1}}-\frac{\gamma_{k}}{1-\beta_{k}} \mid\left(\left\|P_{V I\left(\cap \cap_{i=1}^{N} \operatorname{Fix}\left(T_{i}\right), B\right)}\left(z_{k}-\lambda A z_{k}\right)\right\|+\bar{\epsilon}_{k}\right) \\
& +\frac{\gamma_{k+1}}{1-\beta_{k+1}}\left\|P_{V I\left(\cap \cap_{i=1}^{N} \operatorname{Fix}\left(T_{i}\right), B\right)}\left(z_{k+1}-\lambda A z_{k+1}\right)-P_{V I\left(\cap_{i=1}^{N} \operatorname{Fix}\left(T_{i}\right), B\right)}\left(z_{k}-\lambda A z_{k}\right)\right\| \\
& +\frac{\gamma_{k+1}}{1-\beta_{k+1}}\left(\left\|P_{V I\left(\cap_{i=1}^{N} \operatorname{Fix}\left(T_{i}\right), B\right)}\left(z_{k+1}-\lambda A z_{k+1}\right)-h_{k+1}\right\|\right. \\
& \left.+\left\|P_{V I\left(\cap \cap_{i=1}^{N} \operatorname{Fix}\left(T_{i}\right), B\right)}\left(z_{k}-\lambda A z_{k}\right)-h_{k}\right\|\right)-\left\|x_{k+1}-x_{k}\right\| \\
\leqslant & \left|\frac{\alpha_{k+1}}{1-\beta_{k+1}}-\frac{\alpha_{k}}{1-\beta_{k}}\right|\|u\|+\left|\frac{\gamma_{k+1}}{1-\beta_{k+1}}-\frac{\gamma_{k}}{1-\beta_{k}}\right|\left(\left\|P_{V I\left(\cap_{i=1}^{N} \operatorname{Fix}\left(T_{i}\right), B\right)}\left(z_{k}-\lambda A z_{k}\right)\right\|+\bar{\epsilon}_{k}\right) \\
& +\frac{\gamma_{k+1}}{1-\beta_{k+1}}\left\|z_{k+1}-z_{k}\right\|+\frac{\gamma_{k+1}}{1-\beta_{k+1}}\left(\bar{\epsilon}_{k+1}+\bar{\epsilon}_{k}\right)-\left\|x_{k+1}-x_{k}\right\| .
\end{aligned}
$$

Thus,

$$
\begin{aligned}
& \left\|w_{k+1}-w_{k}\right\|-\left\|x_{k+1}-x_{k}\right\| \\
& \leqslant \\
& \quad\left|\frac{\alpha_{k+1}}{1-\beta_{k+1}}-\frac{\alpha_{k}}{1-\beta_{k}}\right|\|u\|+\left|\frac{\gamma_{k+1}}{1-\beta_{k+1}}-\frac{\gamma_{k}}{1-\beta_{k}}\right|\left(\left\|P_{V I\left(\cap \cap_{i=1}^{N} \operatorname{Fix}\left(T_{i}\right), B\right.}\left(z_{k}-\lambda A z_{k}\right)\right\|+\bar{\epsilon}_{k}\right) \\
& \quad+\frac{\gamma_{k+1}\left(1+\lambda_{k+1} L_{2}\right)}{1-\beta_{k+1}}\left(\left\|v_{k+1}-x_{k+1}\right\|+\left\|x_{k+1}-x_{k}\right\|+\left\|x_{k}-v_{k}\right\|\right) \\
& \quad+\frac{\gamma_{k+1}}{1-\beta_{k+1}}\left[\lambda_{k+1}\left(\left\|B v_{k+1}\right\|+\left\|B y_{k+1}\right\|+\left\|B v_{k}\right\|\right)+\lambda_{k}\left\|B y_{k}\right\|\right]+\frac{\gamma_{k+1}}{1-\beta_{k+1}}\left(\bar{\epsilon}_{k+1}+\bar{\epsilon}_{k}\right)-\left\|x_{k+1}-x_{k}\right\| .
\end{aligned}
$$

Since $\alpha_{k}+\beta_{k}+\gamma_{k}=1, \alpha_{k} \rightarrow 0, \beta_{k} \rightarrow \xi \in\left(0, \frac{1}{2}\right], \bar{\epsilon}_{k} \rightarrow 0, \lambda_{k} \rightarrow 0$, and $\left\|x_{k}-v_{k}\right\| \rightarrow 0$, we deduce from the boundedness of the sequences $\left\{x_{k}\right\},\left\{v_{k}\right\},\left\{y_{k}\right\},\left\{z_{k}\right\}$ that

$$
\limsup _{k \rightarrow \infty}\left(\left\|w_{k+1}-w_{k}\right\|-\left\|x_{k+1}-x_{k}\right\|\right) \leqslant 0
$$

Therefore, in terms of Proposition 3.7, we have $\lim _{k \rightarrow \infty}\left\|w_{k}-x_{k}\right\|=0$ which together with $x_{k+1}=$ $\left(1-\beta_{k}\right) w_{k}+\beta_{k} x_{k}$, yields

$$
\lim _{k \rightarrow \infty}\left\|x_{k+1}-x_{k}\right\|=\lim _{k \rightarrow \infty}\left(1-\beta_{k}\right)\left\|w_{k}-x_{k}\right\|=0 .
$$

Lemma 3.9. Suppose that the conditions (A1) and (H1)-(H4) hold. Then for any $p \in \Omega:=\operatorname{VI}\left(\operatorname{VI}\left(\cap_{i=1}^{N} \operatorname{Fix}\left(T_{i}\right), B\right)\right.$, A) we have

$$
\begin{aligned}
\left\|x_{k+1}-p\right\|^{2} \leqslant & \alpha_{k}\|u-p\|^{2}+\beta_{k}\left\|x_{k}-p\right\|^{2}+\gamma_{k}\left\|v_{k}-p\right\|^{2}+2 \gamma_{k} \bar{\epsilon}_{k}\left\|z_{k}-p\right\| \\
& +\gamma_{k} \bar{\epsilon}_{k}^{2}-\gamma_{k}\left(1-\lambda_{k} L_{2}\right)\left(\left\|v_{k}-y_{k}\right\|^{2}+\left\|y_{k}-z_{k}\right\|^{2}\right) .
\end{aligned}
$$


Moreover, if $\lim _{k \rightarrow \infty}\left\|x_{k}-v_{k}\right\|=0$, then

$$
\lim _{k \rightarrow \infty}\left\|P_{V I\left(\cap \cap_{i=1}^{N} \operatorname{Fix}\left(T_{i}\right), B\right)}\left(z_{k}-\lambda_{k} A z_{k}\right)-z_{k}\right\|=\lim _{k \rightarrow \infty}\left\|P_{V I\left(\cap_{i=1}^{N} \operatorname{Fix}\left(T_{i}\right), B\right)}\left(y_{k}-\lambda_{k} A y_{k}\right)-y_{k}\right\|=0 .
$$

Proof. By Lemma 3.3, we know that

$$
\left.\lim _{j \rightarrow \infty} x_{k, j}=P_{V I(\cap i=1}^{N} \operatorname{Fix}\left(T_{i}\right), B\right)\left(z_{k}-\lambda A z_{k}\right),
$$

which together with $0<\lambda \leqslant 2 \beta$, inequality (3.1), $\lim _{k \rightarrow \infty} \beta_{k}=\xi \in\left(0, \frac{1}{2}\right]$, and

$$
p \in \Omega:=\operatorname{VI}\left(\operatorname{VI}\left(\cap_{i=1}^{N} \operatorname{Fix}\left(T_{i}\right), B\right), A\right),
$$

implies that

$$
\begin{aligned}
\left\|x_{k+1}-p\right\|^{2}= & \left\|\alpha_{k} u+\beta_{k} x_{k}+\gamma_{k} h_{k}-p\right\|^{2} \\
\leqslant & \alpha_{k}\|u-p\|^{2}+\beta_{k}\left\|x_{k}-p\right\|^{2}+\gamma_{k}\left\|h_{k}-p\right\|^{2} \\
\leqslant & \alpha_{k}\|u-p\|^{2}+\beta_{k}\left\|x_{k}-p\right\|^{2}+\gamma_{k}\left(\left\|P_{V I\left(\cap \cap_{i=1}^{N} \operatorname{Fix}\left(T_{i}\right), B\right)}\left(z_{k}-\lambda A z_{k}\right)-p\right\|+\bar{\epsilon}_{k}\right)^{2} \\
\leqslant & \alpha_{k}\|u-p\|^{2}+\beta_{k}\left\|x_{k}-p\right\|^{2}+\gamma_{k}\left(\left\|(I-\lambda A) z_{k}-(I-\lambda A) p\right\|+\bar{\epsilon}_{k}\right)^{2} \\
\leqslant & \alpha_{k}\|u-p\|^{2}+\beta_{k}\left\|x_{k}-p\right\|^{2}+\gamma_{k}\left(\left\|z_{k}-p\right\|+\bar{\epsilon}_{k}\right)^{2} \\
= & \alpha_{k}\|u-p\|^{2}+\beta_{k}\left\|x_{k}-p\right\|^{2}+\gamma_{k}\left\|z_{k}-p\right\|^{2}+2 \gamma_{k} \bar{\epsilon}_{k}\left\|z_{k}-p\right\|+\gamma_{k} \bar{\epsilon}_{k}^{2} \\
\leqslant & \alpha_{k}\|u-p\|^{2}+\beta_{k}\left\|x_{k}-p\right\|^{2}+2 \gamma_{k} \bar{\epsilon}_{k}\left\|z_{k}-p\right\|+\gamma_{k} \bar{\epsilon}_{k}^{2} \\
& +\gamma_{k}\left(\left\|v_{k}-p\right\|^{2}-\left(1-\lambda_{k} L_{2}\right)\left\|v_{k}-y_{k}\right\|^{2}-\left(1-\lambda_{k} L_{2}\right)\left\|y_{k}-z_{k}\right\|^{2}\right) \\
= & \alpha_{k}\|u-p\|^{2}+\beta_{k}\left\|x_{k}-p\right\|^{2}+\gamma_{k}\left\|v_{k}-p\right\|^{2}+2 \gamma_{k} \bar{\epsilon}_{k}\left\|z_{k}-p\right\|+\gamma_{k} \bar{\epsilon}_{k}^{2} \\
& -\gamma_{k}\left(1-\lambda_{k} L_{2}\right)\left(\left\|v_{k}-y_{k}\right\|^{2}+\left\|y_{k}-z_{k}\right\|^{2}\right) .
\end{aligned}
$$

On the other hand, note that $\left\|v_{k}-u_{k}\right\|=\alpha_{k}\left\|v \mathcal{A} u_{k}\right\|$. Since $\mathcal{A}$ is $L_{1}$-Lipschitz continuous and $\left\{\mathfrak{u}_{k}\right\}$ is bounded, we know that $\left\{\mathcal{A} u_{k}\right\}$ is bounded. Hence, it follows that

$$
\lim _{k \rightarrow \infty}\left\|v_{k}-u_{k}\right\|=0,
$$

which together with the assumption $\lim _{k \rightarrow \infty}\left\|x_{k}-v_{k}\right\|=0$, yields

$$
\lim _{k \rightarrow \infty}\left\|x_{k}-u_{k}\right\|=0
$$

Also, from (3.16) it is found that

$$
\begin{aligned}
\gamma_{k}\left(1-\lambda_{k} L_{2}\right)\left(\left\|v_{k}-y_{k}\right\|^{2}+\left\|y_{k}-z_{k}\right\|^{2}\right) \leqslant & \alpha_{k}\|u-p\|^{2}+\beta_{k}\left\|x_{k}-p\right\|^{2}+\gamma_{k}\left\|v_{k}-p\right\|^{2}-\left\|x_{k+1}-p\right\|^{2} \\
& +2 \gamma_{k} \bar{\epsilon}_{k}\left\|z_{k}-p\right\|+\gamma_{k} \bar{\epsilon}_{k}^{2} \\
= & \alpha_{k}\left(\|u-p\|^{2}-\left\|x_{k+1}-p\right\|^{2}\right)+\beta_{k}\left(\left\|x_{k}-p\right\|^{2}-\left\|x_{k+1}-p\right\|^{2}\right) \\
& +\gamma_{k}\left(\left\|v_{k}-p\right\|^{2}-\left\|x_{k+1}-p\right\|^{2}\right)+2 \gamma_{k} \bar{\epsilon}_{k}\left\|z_{k}-p\right\|+\gamma_{k} \bar{\epsilon}_{k}^{2} \\
\leqslant & \alpha_{k}\|u-p\|^{2}+\beta_{k}\left\|x_{k}-x_{k+1}\right\|\left(\left\|x_{k}-p\right\|+\left\|x_{k+1}-p\right\|\right) \\
& +\gamma_{k}\left\|v_{k}-x_{k+1}\right\|\left(\left\|v_{k}-p\right\|+\left\|x_{k+1}-p\right\|\right)+2 \gamma_{k} \bar{\epsilon}_{k}\left\|z_{k}-p\right\|+\gamma_{k} \bar{\epsilon}_{k}^{2} \\
\leqslant & \alpha_{k}\|u-p\|^{2}+\beta_{k}\left\|x_{k}-x_{k+1}\right\|\left(\left\|x_{k}-p\right\|+\left\|x_{k+1}-p\right\|\right) \\
& +\gamma_{k}\left(\left\|v_{k}-x_{k}\right\|+\left\|x_{k}-x_{k+1}\right\|\right)\left(\left\|v_{k}-p\right\|+\left\|x_{k+1}-p\right\|\right) \\
& +2 \gamma_{k} \bar{\epsilon}_{k}\left\|z_{k}-p\right\|+\gamma_{k} \bar{\epsilon}_{k}^{2} .
\end{aligned}
$$

Since $\alpha_{k}+\beta_{k}+\gamma_{k}=1, \alpha_{k} \rightarrow 0, \beta_{k} \rightarrow \xi \in\left(0, \frac{1}{2}\right], \bar{\epsilon}_{k} \rightarrow 0, \lambda_{k} \rightarrow 0,\left\|x_{k}-v_{k}\right\| \rightarrow 0$, and $\left\|x_{k+1}-x_{k}\right\| \rightarrow 0$ (due to Lemma 3.8 (ii)), we deduce from the boundedness of $\left\{x_{k}\right\},\left\{v_{k}\right\}$, and $\left\{z_{k}\right\}$ that

$$
\lim _{k \rightarrow \infty}\left\|v_{k}-y_{k}\right\|=0 \text { and } \lim _{k \rightarrow \infty}\left\|y_{k}-z_{k}\right\|=0,
$$


which together with $\lim _{k \rightarrow \infty}\left\|x_{k}-v_{k}\right\|=0$, imply that

$$
\lim _{k \rightarrow \infty}\left\|x_{k}-y_{k}\right\|=0 \text { and } \lim _{k \rightarrow \infty}\left\|x_{k}-z_{k}\right\|=0 \text {. }
$$

Again by Proposition 2.1 (iii) and Lemma 3.3 we have

$$
\begin{aligned}
& \left\|P_{\mathrm{VI}\left(\cap_{i=1}^{N} \operatorname{Fix}\left(T_{i}\right), B\right)}\left(y_{k}-\lambda A y_{k}\right)-x_{k+1}\right\| \\
& \leqslant\left\|P_{\mathrm{VI}\left(\cap_{i=1}^{N} \operatorname{Fix}\left(T_{i}\right), \mathrm{B}\right)}\left(y_{k}-\lambda A y_{k}\right)-P_{\mathrm{VI}\left(\cap_{i=1}^{N} \operatorname{Fix}\left(T_{i}\right), B\right)}\left(z_{k}-\lambda A z_{k}\right)\right\| \\
& +\left\|P_{\mathrm{VI}\left(\cap_{i=1}^{N} \operatorname{Fix}\left(\mathrm{T}_{\mathrm{i}}\right), \mathrm{B}\right)}\left(z_{\mathrm{k}}-\lambda \mathrm{A} z_{\mathrm{k}}\right)-\mathrm{x}_{\mathrm{k}+1}\right\| \\
& \left.\leqslant\left(1+\lambda \mathrm{L}_{1}\right)\left\|y_{k}-z_{k}\right\|+\alpha_{k} \| P_{V I(\cap N=1}^{N} \operatorname{Fix}\left(T_{i}\right), B\right)\left(z_{k}-\lambda A z_{k}\right)-u \|
\end{aligned}
$$

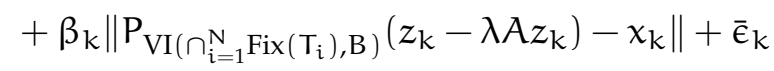

$$
\begin{aligned}
& \leqslant\left(1+\lambda \mathrm{L}_{1}\right)\left\|\mathrm{y}_{\mathrm{k}}-z_{\mathrm{k}}\right\|+\alpha_{\mathrm{k}}\left\|\mathrm{P}_{\mathrm{VI}\left(\cap_{i=1}^{N} \operatorname{Fix}\left(\mathrm{T}_{i}\right), \mathrm{B}\right)}\left(z_{\mathrm{k}}-\lambda \mathrm{A} z_{\mathrm{k}}\right)-\mathrm{u}\right\|+\bar{\epsilon}_{\mathrm{k}} \\
& +\beta_{k}\left\|P_{V I\left(\cap_{i=1}^{N} \operatorname{Fix}\left(T_{i}\right), B\right)}\left(z_{k}-\lambda A z_{k}\right)-P_{V I\left(\cap_{i=1}^{N} \operatorname{Fix}\left(T_{i}\right), B\right)}\left(y_{k}-\lambda A y_{k}\right)\right\| \\
& +\beta_{k}\left\|P_{V I\left(\cap_{i=1}^{N} \operatorname{Fix}\left(T_{i}\right), B\right)}\left(y_{k}-\lambda A y_{k}\right)-y_{k}\right\|+\beta_{k}\left\|y_{k}-x_{k}\right\| \\
& \leqslant\left(1+\lambda \mathrm{L}_{1}\right)\left\|\mathrm{y}_{\mathrm{k}}-z_{\mathrm{k}}\right\|+\alpha_{\mathrm{k}}\left\|\mathrm{P}_{\mathrm{VI}\left(\cap_{i=1}^{N} \operatorname{Fix}\left(\mathrm{T}_{i}\right), \mathrm{B}\right)}\left(z_{\mathrm{k}}-\lambda \mathrm{A} z_{\mathrm{k}}\right)-\mathrm{u}\right\|+\bar{\epsilon}_{\mathrm{k}} \\
& +\beta_{k}\left(1+\lambda L_{1}\right)\left\|z_{k}-y_{k}\right\|+\beta_{k}\left\|P_{V I\left(\cap_{i=1}^{N} \operatorname{Fix}\left(T_{i}\right), B\right)}\left(y_{k}-\lambda A y_{k}\right)-y_{k}\right\|+\beta_{k}\left\|y_{k}-x_{k}\right\| \text {. }
\end{aligned}
$$

Consequently, from (3.19), we have

$$
\begin{aligned}
\| & P_{\mathrm{VI}\left(\cap_{i=1}^{N} \operatorname{Fix}\left(T_{i}\right), B\right)}\left(y_{k}-\lambda A y_{k}\right)-y_{k} \| \\
\leqslant & \left\|P_{V I\left(\cap \cap_{i=1}^{N} \operatorname{Fix}\left(T_{i}\right), B\right)}\left(y_{k}-\lambda A y_{k}\right)-x_{k+1}\right\|+\left\|x_{k+1}-x_{k}\right\|+\left\|x_{k}-y_{k}\right\| \\
\leqslant & \left(1+\lambda L_{1}\right)\left\|y_{k}-z_{k}\right\|+\alpha_{k}\left\|P_{V I\left(\cap \cap_{i=1}^{N} \operatorname{Fix}\left(T_{i}\right), B\right)}\left(z_{k}-\lambda A z_{k}\right)-u\right\|+\bar{\epsilon}_{k} \\
& +\beta_{k}\left(1+\lambda L_{1}\right)\left\|z_{k}-y_{k}\right\|+\beta_{k}\left\|P_{V I\left(\cap_{i=1}^{N} \operatorname{Fix}\left(T_{i}\right), B\right)}\left(y_{k}-\lambda A y_{k}\right)-y_{k}\right\|+\beta_{k}\left\|y_{k}-x_{k}\right\| \\
& +\left\|x_{k+1}-x_{k}\right\|+\left\|x_{k}-y_{k}\right\| \\
= & \left(1+\beta_{k}\right)\left(1+\lambda L_{1}\right)\left\|y_{k}-z_{k}\right\|+\alpha_{k}\left\|P_{V I\left(\cap \cap_{i=1}^{N} \operatorname{Fix}\left(T_{i}\right), B\right)}\left(z_{k}-\lambda A z_{k}\right)-u\right\|+\bar{\epsilon}_{k} \\
& +\beta_{k}\left\|P_{V I\left(\cap \cap_{i=1}^{N} \operatorname{Fix}\left(T_{i}\right), B\right)}\left(y_{k}-\lambda A y_{k}\right)-y_{k}\right\|+\left(1+\beta_{k}\right)\left\|y_{k}-x_{k}\right\|+\left\|x_{k+1}-x_{k}\right\|,
\end{aligned}
$$

which immediately yields

$$
\begin{aligned}
& \left\|P_{\mathrm{VI}\left(\cap_{i=1}^{N} \operatorname{Fix}\left(T_{i}\right), B\right)}\left(y_{k}-\lambda A y_{k}\right)-y_{k}\right\| \\
& \leqslant \\
& \leqslant \frac{1+\beta_{k}}{1-\beta_{k}}\left(1+\lambda L_{1}\right)\left\|y_{k}-z_{k}\right\|+\frac{\alpha_{k}}{1-\beta_{k}}\left\|P_{V I\left(\cap_{i=1}^{N} \operatorname{Fix}\left(T_{i}\right), B\right)}\left(z_{k}-\lambda A z_{k}\right)-u\right\|+\frac{\bar{\epsilon}_{k}}{1-\beta_{k}} \\
& \quad+\frac{1+\beta_{k}}{1-\beta_{k}}\left\|y_{k}-x_{k}\right\|+\frac{1}{1-\beta_{k}}\left\|x_{k+1}-x_{k}\right\| .
\end{aligned}
$$

Since $\alpha_{k}+\beta_{k}+\gamma_{k}=1, \alpha_{k} \rightarrow 0, \beta_{k} \rightarrow \xi \in\left(0, \frac{1}{2}\right], \bar{\epsilon}_{k} \rightarrow 0,\left\|y_{k}-z_{k}\right\| \rightarrow 0,\left\|x_{k}-y_{k}\right\| \rightarrow 0$, and $\left\|x_{k+1}-x_{k}\right\| \rightarrow 0$ (due to Lemma 3.8 (ii), (3.17), and (3.18)), we conclude that

$$
\left.\lim _{k \rightarrow \infty} \| P_{V I(\cap i=1}^{N} \operatorname{Fix}\left(T_{i}\right), B\right)\left(y_{k}-\lambda A y_{k}\right)-y_{k} \|=0 .
$$

From Proposition 2.1 (iii), it follows that

$$
\begin{aligned}
\left\|\mathrm{P}_{\mathrm{VI}\left(\cap_{i=1}^{N} \operatorname{Fix}\left(T_{i}\right), \mathrm{B}\right)}\left(z_{k}-\lambda A z_{k}\right)-z_{k}\right\| \leqslant & \left\|\mathrm{P}_{\mathrm{VI}\left(\cap_{i=1}^{N} \operatorname{Fix}\left(\mathrm{T}_{i}\right), \mathrm{B}\right)}\left(z_{k}-\lambda A z_{k}\right)-\mathrm{P}_{\mathrm{VI}\left(\cap_{i=1}^{N} \operatorname{Fix}\left(\mathrm{T}_{i}\right), \mathrm{B}\right)}\left(y_{k}-\lambda A y_{k}\right)\right\| \\
& +\left\|\mathrm{P}_{\mathrm{VI}\left(\cap_{i=1}^{N} \operatorname{Fix}\left(\mathrm{T}_{i}\right), \mathrm{B}\right)}\left(y_{k}-\lambda A y_{k}\right)-y_{k}\right\|+\left\|y_{k}-z_{k}\right\| \\
\leqslant & \left(1+\lambda \mathrm{L}_{1}\right)\left\|z_{k}-y_{k}\right\|+\left\|\mathrm{P}_{\mathrm{VI}\left(\cap_{i=1}^{N} \operatorname{Fix}\left(\mathrm{T}_{i}\right), \mathrm{B}\right)}\left(y_{k}-\lambda A y_{k}\right)-y_{k}\right\|+\left\|y_{k}-z_{k}\right\| \\
\leqslant & \left\|P_{\mathrm{VI}\left(\cap_{i=1}^{N} \operatorname{Fix}\left(\mathrm{T}_{i}\right), B\right)}\left(y_{k}-\lambda A y_{k}\right)-y_{k}\right\|+\left(2+\lambda \mathrm{L}_{1}\right)\left\|y_{k}-z_{k}\right\| .
\end{aligned}
$$


Utilizing the last inequality we obtain from (3.17) and (3.20) that

$$
\lim _{k \rightarrow \infty}\left\|P_{V I\left(\cap \cap_{i=1}^{N} \operatorname{Fix}\left(T_{i}\right), B\right)}\left(z_{k}-\lambda A z_{k}\right)-z_{k}\right\|=0 .
$$

Theorem 3.10. Suppose that the conditions (A1) and (H1)-(H4) hold. Then the two sequences $\left\{x_{k}\right\}$ and $\left\{z_{k}\right\}$ in Algorithm 3.2 converge strongly to the same point $x^{*} \in \Omega:=\operatorname{VI}\left(\operatorname{VI}\left(\cap_{i=1}^{N} \operatorname{Fix}\left(T_{i}\right), B\right), A\right)$ provided $\left\|x_{k}-v_{k}\right\|=$ $\mathrm{o}\left(\alpha_{\mathrm{k}}\right)$, which is a unique solution to the VIP

$$
\left\langle\mathcal{A} x^{*}, p-x^{*}\right\rangle \geqslant 0, \quad \forall p \in \Omega .
$$

Proof. Note that Lemma 3.5 shows the boundedness of $\left\{x_{k}\right\}$. Since $H$ is reflexive, there is at least a weak convergence subsequence of $\left\{x_{k}\right\}$. First, let us assert that $\omega_{w}\left(x_{k}\right) \subset \Omega$. As a matter of fact, take an arbitrary $w \in \omega_{w}\left(x_{k}\right)$. Then there exists a subsequence $\left\{x_{k_{i}}\right\}$ of $\left\{x_{k}\right\}$ such that $x_{k_{i}} \rightarrow w$. From (3.18), we know that $y_{k_{i}} \rightarrow w$. It is easy to see that the mapping $\mathrm{P}_{\mathrm{VI}\left(\cap_{i=1}^{N} \operatorname{Fix}\left(\mathrm{T}_{i}\right), \mathrm{B}\right)}(\mathrm{I}-\lambda A): \mathrm{H} \rightarrow \operatorname{VI}\left(\cap_{i=1}^{N} \operatorname{Fix}\left(\mathrm{T}_{i}\right), \mathrm{B}\right) \subset$ $H$ is nonexpansive because $P_{V I\left(\cap \cap_{i=1}^{N} \operatorname{Fix}\left(T_{i}\right), B\right)}$ is nonexpansive and $I-\lambda A$ is nonexpansive for $\beta$-inverse strongly monotone mapping $A$ with $0<\lambda \leqslant 2 \beta$. So, utilizing Lemma 2.6 and (3.20), we obtain

$$
w=\mathrm{P}_{\mathrm{VI}\left(\cap_{i=1}^{N} \operatorname{Fix}\left(\mathrm{T}_{i}\right), \mathrm{B}\right)}(w-\lambda A w),
$$

which leads to $w \in \operatorname{VI}\left(\operatorname{VI}\left(\cap_{i=1}^{N} \operatorname{Fix}\left(T_{i}\right), B\right), A\right)=: \Omega$. Thus, the assertion is valid.

Also, note that $\mathcal{A}$ is $\eta$-strongly monotone and $\mathrm{L}_{1}$-Lipschitz continuous on $\mathrm{H}$. Thus, by Lemma 2.5 (iv), we know that there exists a unique solution $x^{*} \in \Omega:=\operatorname{VI}\left(\operatorname{VI}\left(\cap_{i=1}^{N} \operatorname{Fix}\left(T_{i}\right), B\right), A\right)$ to the VIP

$$
\left\langle\mathcal{A} x^{*}, p-x^{*}\right\rangle \geqslant 0, \quad \forall p \in \Omega .
$$

Next, let us show that $x_{k} \rightarrow x^{*}$. Indeed, take an arbitrary $p \in \Omega:=\operatorname{VI}\left(\operatorname{VI}\left(\cap_{i=1}^{N} \operatorname{Fix}\left(T_{i}\right), B\right), A\right)$. Utilizing the monotonicity of $\mathcal{A}$, we obtain from Algorithm 3.2 that for all $k \geqslant 0$,

$$
\begin{aligned}
\left\|v_{k}-p\right\|^{2} & =\left\|u_{k}-\alpha_{k} v \mathcal{A} u_{k}-p\right\|^{2} \\
& \leqslant\left\|\left(u_{k}-p\right)-\alpha_{k} v \mathcal{A} u_{k}\right\|^{2} \\
& =\left\|u_{k}-p\right\|^{2}+2 \alpha_{k} v\left\langle\mathcal{A} u_{k}, p-u_{k}\right\rangle+v^{2} \alpha_{k}^{2}\left\|\mathcal{A} u_{k}\right\|^{2} \\
& \leqslant\left\|u_{k}-p\right\|^{2}+2 \alpha_{k} v\left\langle\mathcal{A} p, p-u_{k}\right\rangle+v^{2} \alpha_{k}^{2}\left\|\mathcal{A} u_{k}\right\|^{2} \\
& =\left\|u_{k}-p\right\|^{2}+2 \alpha_{k} v\left\langle\mathcal{A p}, p-x_{k}+x_{k}-u_{k}\right\rangle+v^{2} \alpha_{k}^{2}\left\|\mathcal{A} u_{k}\right\|^{2} \\
& \leqslant\left\|u_{k}-p\right\|^{2}+2 \alpha_{k} v\left(\left\langle\mathcal{A p}, p-x_{k}\right\rangle+\|\mathcal{A} p\|\left\|x_{k}-u_{k}\right\|\right)+v^{2} \alpha_{k}^{2}\left\|\mathcal{A} u_{k}\right\|^{2} .
\end{aligned}
$$

Also, utilizing the convexity of $\|\cdot\|^{2}$, we obtain from Algorithm 3.2 that

$$
\begin{aligned}
\left\|u_{k}-p\right\|^{2} & =\left\|\beta_{k} x_{k}+\left(1-\beta_{k}\right) T^{\left(\lambda_{k}, \mu\right)} u_{k}-p\right\|^{2} \\
& =\left\|\beta_{k}\left(x_{k}-p\right)+\left(1-\beta_{k}\right)\left(T^{\left(\lambda_{k}, \mu\right)} u_{k}-p\right)\right\|^{2} \\
& \leqslant \beta_{k}\left\|x_{k}-p\right\|^{2}+\left(1-\beta_{k}\right)\left\|T^{\left(\lambda_{k}, \mu\right)} u_{k}-p\right\|^{2} .
\end{aligned}
$$

Utilizing Lemma 2.10, we get

$$
\begin{aligned}
\left\|T^{\left(\lambda_{k}, \mu\right)} u_{k}-p\right\| & =\left\|T^{\left(\lambda_{k}, \mu\right)} u_{k}-T^{\left(\lambda_{k}, \mu\right)} p+T^{\left(\lambda_{k}, \mu\right)} p-p\right\| \\
& \leqslant\left\|T^{\left(\lambda_{k}, \mu\right)} u_{k}-T^{\left(\lambda_{k}, \mu\right)} p\right\|+\left\|T^{\left(\lambda_{k}, \mu\right)} p-p\right\| \\
& \leqslant\left(1-\lambda_{k} \tau\right)\left\|u_{k}-p\right\|+\lambda_{k} \mu\|F p\|,
\end{aligned}
$$


which implies that

$$
\left\|T^{\left(\lambda_{k}, \mu\right)} \mathfrak{u}_{k}-p\right\|^{2} \leqslant\left(1-\lambda_{k} \tau\right)\left\|u_{k}-p\right\|^{2}+\lambda_{k} \frac{\mu^{2}\|F p\|^{2}}{\tau}
$$

This together with (3.23) yields

$$
\begin{aligned}
\left\|u_{k}-p\right\|^{2} & \leqslant \beta_{k}\left\|x_{k}-p\right\|^{2}+\left(1-\beta_{k}\right)\left\|T^{\left(\lambda_{k}, \mu\right)} u_{k}-p\right\|^{2} \\
& \leqslant \beta_{k}\left\|x_{k}-p\right\|^{2}+\left(1-\beta_{k}\right)\left[\left(1-\lambda_{k} \tau\right)\left\|u_{k}-p\right\|^{2}+\lambda_{k} \frac{\mu^{2}\|F p\|^{2}}{\tau}\right] \\
& \leqslant \beta_{k}\left\|x_{k}-p\right\|^{2}+\left(1-\beta_{k}\right)\left\|u_{k}-p\right\|^{2}+\left(1-\beta_{k}\right) \lambda_{k} \frac{\mu^{2}\|F p\|^{2}}{\tau}
\end{aligned}
$$

and so

$$
\left\|u_{k}-p\right\|^{2} \leqslant\left\|x_{k}-p\right\|^{2}+\left(1-\beta_{k}\right) \frac{\lambda_{k}}{\beta_{k}} \cdot \frac{\mu^{2}\|F p\|^{2}}{\tau} \leqslant\left\|x_{k}-p\right\|^{2}+\lambda_{k} \cdot \frac{\mu^{2}\|F p\|^{2}}{\tau a} .
$$

Combining (3.22) and (3.24), we get

$$
\begin{aligned}
\left\|v_{k}-p\right\|^{2} & \leqslant\left\|u_{k}-p\right\|^{2}+2 \alpha_{k} v\left(\left\langle\mathcal{A p}, p-x_{k}\right\rangle+\|\mathcal{A} p\|\left\|x_{k}-u_{k}\right\|\right)+v^{2} \alpha_{k}^{2}\left\|\mathcal{A} u_{k}\right\|^{2} \\
& \leqslant\left\|x_{k}-p\right\|^{2}+\lambda_{k} \cdot \frac{\mu^{2}\|F p\|^{2}}{\tau a}+2 v \alpha_{k}\left(\left\langle\mathcal{A p}, p-x_{k}\right\rangle+\|\mathcal{A} p\|\left\|x_{k}-u_{k}\right\|\right)+v^{2} \alpha_{k}^{2}\left\|\mathcal{A} u_{k}\right\|^{2},
\end{aligned}
$$

which immediately leads to

$$
\begin{aligned}
0 & \leqslant\left\|x_{k}-p\right\|^{2}-\left\|v_{k}-p\right\|^{2}+\lambda_{k} \cdot \frac{\mu^{2}\|\mathrm{~F} p\|^{2}}{\tau a}+2 \alpha_{k} v\left(\left\langle\mathcal{A} p, p-x_{k}\right\rangle+\|\mathcal{A} p\|\left\|x_{k}-u_{k}\right\|\right)+v^{2} \alpha_{k}^{2}\left\|\mathcal{A} u_{k}\right\|^{2} \\
& \leqslant\left\|x_{k}-v_{k}\right\|\left(\left\|x_{k}-p\right\|+\left\|v_{k}-p\right\|\right)+\lambda_{k} \cdot \frac{\mu^{2}\|\mathrm{~F} p\|^{2}}{\tau a}+2 \alpha_{k} v\left(\left\langle\mathcal{A p}, p-x_{k}\right\rangle+\|\mathcal{A} p\|\left\|x_{k}-u_{k}\right\|\right)+v^{2} \alpha_{k}^{2}\left\|\mathcal{A} u_{k}\right\|^{2} .
\end{aligned}
$$

That is,

$$
\begin{aligned}
0 \leqslant & \frac{\left\|x_{k}-v_{k}\right\|}{2 \alpha_{k} v}\left(\left\|x_{k}-p\right\|+\left\|v_{k}-p\right\|\right)+\frac{\lambda_{k}}{\alpha_{k}} \cdot \frac{\mu^{2}\|F p\|^{2}}{2 v \tau a}+\left\langle\mathcal{A p}, p-x_{k}\right\rangle \\
& +\|\mathcal{A} p\|\left\|x_{k}-u_{k}\right\|+\frac{v \alpha_{k}}{2}\left\|\mathcal{A} u_{k}\right\|^{2} .
\end{aligned}
$$

Since for any $w \in \omega_{w}\left(x_{k}\right)$ there exists a subsequence $\left\{x_{k_{i}}\right\}$ of $\left\{x_{k}\right\}$ such that $x_{k_{i}} \rightarrow w$, we deduce from (3.25), $\alpha_{k} \rightarrow 0,\left\|x_{k}-u_{k}\right\| \rightarrow 0, \lambda_{k}=o\left(\alpha_{k}\right)$ and $\left\|x_{k}-v_{k}\right\|=o\left(\alpha_{k}\right)$ that for all $p \in \operatorname{VI}\left(\operatorname{VI}\left(\cap_{i=1}^{N} \operatorname{Fix}\left(T_{i}\right), B\right), A\right)$

$$
\begin{aligned}
0 \leqslant & \lim _{i \rightarrow \infty}\left\{\frac{\left\|x_{k_{i}}-v_{k_{i}}\right\|}{2 \alpha_{k_{i}} v}\left(\left\|x_{k_{i}}-p\right\|+\left\|v_{k_{i}}-p\right\|\right)+\frac{\lambda_{k_{i}}}{\alpha_{k_{i}}} \cdot \frac{\mu^{2}\|F p\|^{2}}{2 v \tau a}+\left\langle\mathcal{A p}, p-x_{k_{i}}\right\rangle\right. \\
& \left.+\|\mathcal{A} p\|\left\|x_{k_{i}}-u_{k_{i}}\right\|+\frac{v \alpha_{k_{i}}}{2}\left\|\mathcal{A} u_{k_{i}}\right\|^{2}\right\} \\
= & \lim _{i \rightarrow \infty}\left\langle\mathcal{A} p, p-x_{k_{i}}\right\rangle=\langle\mathcal{A} p, p-w\rangle .
\end{aligned}
$$

Thus, by Lemma 2.5 (i), we know that

$$
\langle\mathcal{A} w, p-w\rangle \geqslant 0, \quad \forall p \in \Omega,
$$

that is, $w$ is a solution of VIP (3.21). By the uniqueness of solutions of VIP (3.21), we get $w=x^{*}$, which hence implies that $\omega_{w}\left(x_{k}\right)=\left\{x^{*}\right\}$. Therefore, it is known that $\left\{x_{k}\right\}$ converges weakly to the unique solution $x^{*} \in \Omega:=\operatorname{VI}\left(\operatorname{VI}\left(\cap_{i=1}^{N} \operatorname{Fix}\left(T_{i}\right), B\right), A\right)$ of $\operatorname{VIP}(3.21)$. 
Finally, let us show that $\left\|x_{k}-x^{*}\right\| \rightarrow 0$ as $k \rightarrow \infty$. Indeed, in terms of Algorithm 3.3 and Lemma 2.4, we conclude from (3.3) and the $\beta$-inverse-strong monotonicity of $A$ with $0<\lambda \leqslant 2 \beta$, that

$$
\begin{aligned}
\left\|x_{k+1}-x^{*}\right\|^{2}= & \left\|\alpha_{k} u+\beta_{k} x_{k}+\gamma_{k} h_{k}-x^{*}\right\|^{2} \\
\leqslant & \left\|\beta_{k}\left(x_{k}-x^{*}\right)+\gamma_{k}\left(h_{k}-x^{*}\right)\right\|^{2}+2 \alpha_{k}\left\langle u-x^{*}, x_{k+1}-x^{*}\right\rangle \\
\leqslant & \beta_{k}\left\|x_{k}-x^{*}\right\|^{2}+\gamma_{k}\left\|h_{k}-x^{*}\right\|^{2}+2 \alpha_{k}\left\langle u-x^{*}, x_{k+1}-x^{*}\right\rangle \\
\leqslant & \gamma_{k}\left(\left\|P_{V I}\left(\cap_{i=1}^{N} \operatorname{Fix}\left(T_{i}\right), B\right)\left(z_{k}-\lambda A z_{k}\right)-P_{V I\left(\cap \cap_{i=1}^{N} \operatorname{Fix}\left(T_{i}\right), B\right)}\left(x^{*}-\lambda A x^{*}\right)\right\|+\bar{\epsilon}_{k}\right)^{2} \\
& +\beta_{k}\left\|x_{k}-x^{*}\right\|^{2}+2 \alpha_{k}\left\langle u-x^{*}, x_{k+1}-x^{*}\right\rangle \\
\leqslant & \beta_{k}\left\|x_{k}-x^{*}\right\|^{2}+\gamma_{k}\left(\left\|(I-\lambda A) z_{k}-(I-\lambda A) x^{*}\right\|+\bar{\epsilon}_{k}\right)^{2}+2 \alpha_{k}\left\langle u-x^{*}, x_{k+1}-x^{*}\right\rangle \\
= & \beta_{k}\left\|x_{k}-x^{*}\right\|^{2}+\gamma_{k}\left\|z_{k}-x^{*}\right\|^{2}+\gamma_{k} \bar{\epsilon}_{k}\left(2\left\|z_{k}-x^{*}\right\|+\bar{\epsilon}_{k}\right)+2 \alpha_{k}\left\langle u-x^{*}, x_{k+1}-x^{*}\right\rangle \\
\leqslant & \beta_{k}\left\|x_{k}-x^{*}\right\|^{2}+\gamma_{k}\left\|v_{k}-x^{*}\right\|^{2}+\gamma_{k} \bar{\epsilon}_{k}\left(2\left\|z_{k}-x^{*}\right\|+\bar{\epsilon}_{k}\right)+2 \alpha_{k}\left\langle u-x^{*}, x_{k+1}-x^{*}\right\rangle \\
= & \beta_{k}\left\|x_{k}-x^{*}\right\|^{2}+\gamma_{k}\left(\left\|x_{k}-x^{*}\right\|^{2}+2\left\langle x_{k}-x^{*}, v_{k}-x_{k}\right\rangle+\left\|v_{k}-x_{k}\right\|^{2}\right) \\
& +\gamma_{k} \bar{\epsilon}_{k}\left(2\left\|z_{k}-x^{*}\right\|+\bar{\epsilon}_{k}\right)+2 \alpha_{k}\left\langle u-x^{*}, x_{k+1}-x^{*}\right\rangle \\
\leqslant & \beta_{k}\left\|x_{k}-x^{*}\right\|^{2}+\gamma_{k}\left(\left\|x_{k}-x^{*}\right\|^{2}+\left\|v_{k}-x_{k}\right\|\left(2\left\|x_{k}-x^{*}\right\|+\left\|v_{k}-x_{k}\right\|\right)\right) \\
& +\gamma_{k} \bar{\epsilon}_{k}\left(2\left\|z_{k}-x^{*}\right\|+\bar{\epsilon}_{k}\right)+2 \alpha_{k}\left\langle u-x^{*}, x_{k+1}-x^{*}\right\rangle \\
\leqslant & \left(1-\alpha_{k}\right)\left\|x_{k}-x^{*}\right\|^{2}+\left\|v_{k}-x_{k}\right\|\left(2\left\|x_{k}-x^{*}\right\|+\left\|v_{k}-x_{k}\right\|\right) \\
& +\bar{\epsilon}_{k}\left(2\left\|z_{k}-x^{*}\right\|+\bar{\epsilon}_{k}\right)+2 \alpha_{k}\left\langle u-x^{*}, x_{k+1}-x^{*}\right\rangle \\
= & \left(1-\alpha_{k}\right)\left\|x_{k}-x^{*}\right\|^{2}+\alpha_{k}\left[\frac{\left\|v_{k}-x_{k}\right\|}{\alpha_{k}}\left(2\left\|x_{k}-x^{*}\right\|+\left\|v_{k}-x_{k}\right\|\right)\right. \\
& \left.+2\left\langle u-x^{*}, x_{k+1}-x^{*}\right\rangle\right]+\bar{\epsilon}_{k}\left(2\left\|z_{k}-x^{*}\right\|+\bar{\epsilon}_{k}\right) .
\end{aligned}
$$

Since $\alpha_{k} \rightarrow 0,\left\|x_{k}-v_{k}\right\|=o\left(\alpha_{k}\right), \sum_{k=0}^{\infty} \bar{\epsilon}_{k}<\infty$ and $x_{k} \rightarrow x^{*}$, we deduce from the boundedness of $\left\{x_{k}\right\},\left\{v_{k}\right\},\left\{z_{k}\right\}$ that $\sum_{k=0}^{\infty} \bar{\epsilon}_{k}\left(2\left\|z_{k}-x^{*}\right\|+\bar{\epsilon}_{k}\right)<\infty$ and

$$
\limsup _{k \rightarrow \infty}\left[\frac{\left\|v_{k}-x_{k}\right\|}{\alpha_{k}}\left(2\left\|x_{k}-x^{*}\right\|+\left\|v_{k}-x_{k}\right\|\right)+2\left\langle u-x^{*}, x_{k+1}-x^{*}\right\rangle\right] \leqslant 0 .
$$

Therefore, applying Lemma 2.12 to (3.26), we infer from $\sum_{k=0}^{\infty} \alpha_{k}=\infty$ that $\left\|x_{k}-x^{*}\right\| \rightarrow 0$ as $k \rightarrow \infty$. Utilizing (3.18) we also obtain that $\left\|z_{k}-x^{*}\right\| \rightarrow 0$ as $k \rightarrow \infty$. This completes the proof.

Theorem 3.11. Suppose that the conditions (A1) and (H1)-(H4) hold. Then the two sequences $\left\{x_{k}\right\}$ and $\left\{z_{k}\right\}$ in Algorithm 3.2 converge strongly to the same point $x^{*} \in \Omega:=\operatorname{VI}\left(\operatorname{VI}\left(\cap_{i=1}^{N} \operatorname{Fix}\left(T_{i}\right), B\right), A\right)$ provided $\left\|x_{k+1}-x_{k}\right\|+$ $\bar{\epsilon}_{\mathrm{k}}=\mathrm{o}\left(\alpha_{\mathrm{k}}\right)$, which is a unique solution to the VIP

$$
\left\langle(\mathrm{I}+\bar{\xi} v \mathcal{A}) x^{*}-u, p-x^{*}\right\rangle \geqslant 0, \quad \forall p \in \Omega,
$$

where $\bar{\xi}=1-\xi$.

Proof. Assume that the conditions (A1) and (H1)-(H4) hold and that $\left\|x_{k+1}-x_{k}\right\|=o\left(\alpha_{k}\right)$. In this case, it is easy to see that Lemmas 3.3-3.6 hold.

Next, we divide the rest of the proof into several steps.

Step 1. We prove that $\lim _{k \rightarrow \infty}\left\|x_{k}-v_{k}\right\|=0$.

Indeed, take an arbitrary $p \in \Omega:=\operatorname{VI}\left(\operatorname{VI}\left(\cap_{i=1}^{N} \operatorname{Fix}\left(T_{i}\right), B\right), A\right)$. By Lemma 3.3, we know that

$$
\left.\lim _{j \rightarrow \infty} x_{k, j}=P_{V I(\cap i=1}^{N} \operatorname{Fix}\left(T_{i}\right), B\right)\left(z_{k}-\lambda A z_{k}\right) .
$$

From $0<\lambda \leqslant 2 \beta$, inequality (3.1) and $\lim _{k \rightarrow \infty} \beta_{k}=\xi \in\left(0, \frac{1}{2}\right]$, utilizing the same argument as in (3.16), 
we obtain

$$
\begin{aligned}
\left\|x_{k+1}-p\right\|^{2} \leqslant & \alpha_{k}\|u-p\|^{2}+\beta_{k}\left\|x_{k}-p\right\|^{2}+\gamma_{k}\left\|\nu_{k}-p\right\|^{2}+2 \gamma_{k} \bar{\epsilon}_{k}\left\|z_{k}-p\right\|+\gamma_{k} \bar{\epsilon}_{k}^{2} \\
& -\gamma_{k}\left(1-\lambda_{k} L_{2}\right)\left(\left\|v_{k}-y_{k}\right\|^{2}+\left\|y_{k}-z_{k}\right\|^{2}\right) .
\end{aligned}
$$

Utilizing Lemma 2.10, we have from the $\bar{\eta}$-strong monotonicity and $\mathrm{L}_{1}$-Lipschitz continuity of $\mathcal{A}$ that

$$
\begin{aligned}
\left\|v_{k}-p\right\|^{2} & =\left\|S^{\left(\alpha_{k}, v\right)} u_{k}-p\right\|^{2} \\
& \leqslant\left[\left\|S^{\left(\alpha_{k}, v\right)} \mathfrak{u}_{k}-S^{\left(\alpha_{k}, v\right)} p\right\|+\left\|S^{\left(\alpha_{k}, v\right)} p-p\right\|\right]^{2} \\
& \leqslant\left[\left(1-\alpha_{k} \sigma\right)\left\|u_{k}-p\right\|+\alpha_{k} v\|\mathcal{A} p\|\right]^{2} \\
& =\left[\left(1-\alpha_{k} \sigma\right)\left\|u_{k}-p\right\|+\alpha_{k} \sigma \frac{v}{\sigma}\|\mathcal{A} p\|\right]^{2} \\
& \leqslant\left(1-\alpha_{k} \sigma\right)\left\|u_{k}-p\right\|^{2}+\alpha_{k} \frac{v^{2}\|\mathcal{A} p\|^{2}}{\sigma} \\
& \leqslant\left\|u_{k}-p\right\|^{2}+\alpha_{k} \frac{v^{2}\|\mathcal{A} p\|^{2}}{\sigma},
\end{aligned}
$$

where $\sigma:=1-\sqrt{1-v\left(2 \bar{\eta}-v L_{1}^{2}\right)}$.

Also, utilizing Lemma 2.13 (b), we obtain from Algorithm 3.2 that

$$
\begin{aligned}
\left\|\mathfrak{u}_{k}-p\right\|^{2} & =\left\|\beta_{k} x_{k}+\left(1-\beta_{k}\right) T^{\left(\lambda_{k}, \mu\right)} \mathfrak{u}_{k}-p\right\|^{2} \\
& =\left\|\beta_{k}\left(x_{k}-p\right)+\left(1-\beta_{k}\right)\left(T^{\left(\lambda_{k}, \mu\right)} \mathfrak{u}_{k}-p\right)\right\|^{2} \\
& =\beta_{k}\left\|x_{k}-p\right\|^{2}+\left(1-\beta_{k}\right)\left\|T^{\left(\lambda_{k}, \mu\right)} \mathfrak{u}_{k}-p\right\|^{2}-\beta_{k}\left(1-\beta_{k}\right)\left\|x_{k}-T^{\left(\lambda_{k}, \mu\right)} \mathfrak{u}_{k}\right\|^{2} .
\end{aligned}
$$

Utilizing Lemma 2.10, we get

$$
\begin{aligned}
\left\|T^{\left(\lambda_{k}, \mu\right)} u_{k}-p\right\| & =\left\|T^{\left(\lambda_{k}, \mu\right)} u_{k}-T^{\left(\lambda_{k}, \mu\right)} p+T^{\left(\lambda_{k}, \mu\right)} p-p\right\| \\
& \leqslant\left\|T^{\left(\lambda_{k}, \mu\right)} u_{k}-T^{\left(\lambda_{k}, \mu\right)} p\right\|+\left\|T^{\left(\lambda_{k}, \mu\right)} p-p\right\| \\
& \leqslant\left(1-\lambda_{k} \tau\right)\left\|u_{k}-p\right\|+\lambda_{k} \mu\|F p\|,
\end{aligned}
$$

which implies that

$$
\left\|T^{\left(\lambda_{k}, \mu\right)} u_{k}-p\right\|^{2} \leqslant\left(1-\lambda_{k} \tau\right)\left\|u_{k}-p\right\|^{2}+\lambda_{k} \frac{\mu^{2}\|F p\|^{2}}{\tau}
$$

So, it follows that

$$
\begin{aligned}
\left\|u_{k}-p\right\|^{2} & =\beta_{k}\left\|x_{k}-p\right\|^{2}+\left(1-\beta_{k}\right)\left\|T^{\left(\lambda_{k}, \mu\right)} u_{k}-p\right\|^{2}-\beta_{k}\left(1-\beta_{k}\right)\left\|x_{k}-T^{\left(\lambda_{k}, \mu\right)} u_{k}\right\|^{2} \\
& \leqslant \beta_{k}\left\|x_{k}-p\right\|^{2}+\left(1-\beta_{k}\right)\left[\left(1-\lambda_{k} \tau\right)\left\|u_{k}-p\right\|^{2}+\lambda_{k} \frac{\mu^{2}\|F p\|^{2}}{\tau}\right]-\beta_{k}\left(1-\beta_{k}\right)\left\|x_{k}-T^{\left(\lambda_{k}, \mu\right)} u_{k}\right\|^{2} \\
& \leqslant \beta_{k}\left\|x_{k}-p\right\|^{2}+\left(1-\beta_{k}\right)\left\|u_{k}-p\right\|^{2}+\left(1-\beta_{k}\right) \lambda_{k} \frac{\mu^{2}\|F p\|^{2}}{\tau}-\beta_{k}\left(1-\beta_{k}\right)\left\|x_{k}-T^{\left(\lambda_{k}, \mu\right)} u_{k}\right\|^{2},
\end{aligned}
$$

and hence

$$
\begin{aligned}
\left\|u_{k}-p\right\|^{2} & \leqslant\left\|x_{k}-p\right\|^{2}+\left(1-\beta_{k}\right) \frac{\lambda_{k}}{\beta_{k}} \cdot \frac{\mu^{2}\|F p\|^{2}}{\tau}-\left(1-\beta_{k}\right)\left\|x_{k}-T^{\left(\lambda_{k}, \mu\right)} u_{k}\right\|^{2} \\
& \leqslant\left\|x_{k}-p\right\|^{2}+\lambda_{k} \cdot \frac{\mu^{2}\|F p\|^{2}}{\tau a}-\left\|u_{k}-x_{k}\right\|^{2} .
\end{aligned}
$$

Combining (3.28), (3.29), and (3.30), we get

$$
\begin{aligned}
\left\|x_{k+1}-p\right\|^{2} \leqslant & \alpha_{k}\|u-p\|^{2}+\beta_{k}\left\|x_{k}-p\right\|^{2}+\gamma_{k}\left(\left\|u_{k}-p\right\|^{2}+\alpha_{k} \frac{v^{2}\|\mathcal{A} p\|^{2}}{\sigma}\right) \\
& +2 \gamma_{k} \bar{\epsilon}_{k}\left\|z_{k}-p\right\|+\gamma_{k} \bar{\epsilon}_{k}^{2}-\gamma_{k}\left(1-\lambda_{k} L_{2}\right)\left(\left\|v_{k}-y_{k}\right\|^{2}+\left\|y_{k}-z_{k}\right\|^{2}\right)
\end{aligned}
$$




$$
\begin{aligned}
\leqslant & \alpha_{k}\|u-p\|^{2}+\beta_{k}\left\|x_{k}-p\right\|^{2}+\gamma_{k}\left[\left\|x_{k}-p\right\|^{2}+\lambda_{k} \cdot \frac{\mu^{2}\|F p\|^{2}}{\tau a}\right. \\
& \left.-\left\|u_{k}-x_{k}\right\|^{2}+\alpha_{k} \frac{v^{2}\|\mathcal{A} p\|^{2}}{\sigma}\right]+2 \gamma_{k} \bar{\epsilon}_{k}\left\|z_{k}-p\right\|+\gamma_{k} \bar{\epsilon}_{k}^{2} \\
& -\gamma_{k}\left(1-\lambda_{k} L_{2}\right)\left(\left\|v_{k}-y_{k}\right\|^{2}+\left\|y_{k}-z_{k}\right\|^{2}\right) \\
= & \alpha_{k}\|u-p\|^{2}+\left(\beta_{k}+\gamma_{k}\right)\left\|x_{k}-p\right\|^{2}+\gamma_{k}\left(\lambda_{k} \cdot \frac{\mu^{2}\|F p\|^{2}}{\tau a}+\alpha_{k} \frac{v^{2}\|\mathcal{A} p\|^{2}}{\sigma}\right) \\
& -\gamma_{k}\left\|u_{k}-x_{k}\right\|^{2}+2 \gamma_{k} \bar{\epsilon}_{k}\left\|z_{k}-p\right\|+\gamma_{k} \bar{\epsilon}_{k}^{2}-\gamma_{k}\left(1-\lambda_{k} L_{2}\right)\left(\left\|v_{k}-y_{k}\right\|^{2}+\left\|y_{k}-z_{k}\right\|^{2}\right) \\
= & \alpha_{k}\|u-p\|^{2}+\left(1-\alpha_{k}\right)\left\|x_{k}-p\right\|^{2}+\gamma_{k}\left(\lambda_{k} \frac{\mu^{2}\|F p\|^{2}}{\tau a}+\alpha_{k} \frac{v^{2}\|\mathcal{A} p\|^{2}}{\sigma}\right) \\
& +2 \gamma_{k} \bar{\epsilon}_{k}\left\|z_{k}-p\right\|+\gamma_{k} \bar{\epsilon}_{k}^{2}-\gamma_{k}\left\|u_{k}-x_{k}\right\|^{2}-\gamma_{k}\left(1-\lambda_{k} L_{2}\right)\left(\left\|v_{k}-y_{k}\right\|^{2}+\left\|y_{k}-z_{k}\right\|^{2}\right) \\
\leqslant & \alpha_{k}\|u-p\|^{2}+\left\|x_{k}-p\right\|^{2}+\lambda_{k} \frac{\mu^{2}\|F p\|^{2}}{\tau a}+\alpha_{k} \frac{v^{2}\|\mathcal{A} p\|^{2}}{\sigma} \\
& +2 \bar{\epsilon}_{k}\left\|z_{k}-p\right\|+\bar{\epsilon}_{k}^{2}-\gamma_{k}\left\|u_{k}-x_{k}\right\|^{2}-\gamma_{k}\left(1-\lambda_{k} L_{2}\right)\left(\left\|v_{k}-y_{k}\right\|^{2}+\left\|y_{k}-z_{k}\right\|^{2}\right),
\end{aligned}
$$

which immediately yields

$$
\begin{aligned}
& \gamma_{k}\left\|u_{k}-x_{k}\right\|^{2}+\gamma_{k}\left(1-\lambda_{k} L_{2}\right)\left(\left\|v_{k}-y_{k}\right\|^{2}+\left\|y_{k}-z_{k}\right\|^{2}\right) \\
& \leqslant \alpha_{k}\|u-p\|^{2}+\left\|x_{k}-p\right\|^{2}-\left\|x_{k+1}-p\right\|^{2}+\lambda_{k} \frac{\mu^{2}\|F p\|^{2}}{\tau a}+\alpha_{k} \frac{v^{2}\|\mathcal{A} p\|^{2}}{\sigma}+2 \bar{\epsilon}_{k}\left\|z_{k}-p\right\|+\bar{\epsilon}_{k}^{2} \\
& \leqslant \alpha_{k}\|u-p\|^{2}+\left\|x_{k}-x_{k+1}\right\|\left(\left\|x_{k}-p\right\|+\left\|x_{k+1}-p\right\|\right)+\lambda_{k} \frac{\mu^{2}\|F p\|^{2}}{\tau a}+\alpha_{k} \frac{v^{2}\|\mathcal{A} p\|^{2}}{\sigma}+2 \bar{\epsilon}_{k}\left\|z_{k}-p\right\|+\bar{\epsilon}_{k}^{2} .
\end{aligned}
$$

Since $\alpha_{k}+\beta_{k}+\gamma_{k}=1, \alpha_{k} \rightarrow 0, \beta_{k} \rightarrow \xi \in\left(0, \frac{1}{2}\right], \bar{\epsilon}_{k} \rightarrow 0, \lambda_{k} \rightarrow 0$, and $\left\|x_{k+1}-x_{k}\right\| \rightarrow 0$ (due to the assumption), we deduce from the boundedness of $\left\{x_{k}\right\}$ and $\left\{z_{k}\right\}$ that

$$
\lim _{k \rightarrow \infty}\left\|u_{k}-x_{k}\right\|=0, \quad \lim _{k \rightarrow \infty}\left\|v_{k}-y_{k}\right\|=0, \quad \text { and } \quad \lim _{k \rightarrow \infty}\left\|y_{k}-z_{k}\right\|=0 .
$$

Note that $\left\|v_{k}-\mathfrak{u}_{k}\right\|=\alpha_{k}\left\|v \mathcal{A} u_{k}\right\|$. Since $\mathcal{A}$ is $\mathrm{L}_{1}$-Lipschitz continuous and $\left\{\mathfrak{u}_{k}\right\}$ is bounded, we know that $\left\{\mathcal{A u}_{\mathrm{k}}\right\}$ is bounded. Hence, it follows that

$$
\lim _{k \rightarrow \infty}\left\|v_{k}-\mathfrak{u}_{k}\right\|=0,
$$

which together with $\lim _{k \rightarrow \infty}\left\|\mathfrak{u}_{k}-x_{k}\right\|=0$, yields

$$
\lim _{k \rightarrow \infty}\left\|v_{k}-x_{k}\right\|=0
$$

This together with (3.31), imply that

$$
\lim _{k \rightarrow \infty}\left\|x_{k}-y_{k}\right\|=0 \text { and } \lim _{k \rightarrow \infty}\left\|x_{k}-z_{k}\right\|=0 .
$$

Step 2. We prove that $\omega_{\mathcal{w}}\left(x_{k}\right) \subset \Omega:=\operatorname{VI}\left(\operatorname{VI}\left(\cap_{i=1}^{N} \operatorname{Fix}\left(T_{i}\right), B\right), A\right)$.

Indeed, from Lemma 3.9 and $\lim _{k \rightarrow \infty}\left\|x_{k}-v_{k}\right\|=0$, we have

$$
\lim _{k \rightarrow \infty}\left\|P_{V I\left(\cap_{i=1}^{N} \operatorname{Fix}\left(T_{i}\right), B\right)}\left(z_{k}-\lambda_{k} A z_{k}\right)-z_{k}\right\|=\lim _{k \rightarrow \infty}\left\|P_{V I\left(\cap_{i=1}^{N} \operatorname{Fix}\left(T_{i}\right), B\right)}\left(y_{k}-\lambda_{k} A y_{k}\right)-y_{k}\right\|=0 .
$$

Utilizing the same argument as in the proof of Theorem 3.10, we obtain that $\omega_{w}\left(x_{k}\right) \subset \Omega$.

Step 3. We prove that $x_{k} \rightarrow x^{*}$ where the $x^{*}$ is a unique solution in $\Omega$ to the VIP (3.27).

Indeed, we define the mapping $\Gamma: \mathrm{H} \rightarrow \mathrm{H}$ as below

$$
\Gamma x=v \mathcal{A} x+\frac{1}{\bar{\xi}}(x-u), \quad \forall x \in H,
$$


where $u \in H$ and $\xi \in\left(0, \frac{1}{2}\right]$. Since $\mathcal{A}$ is $\bar{\eta}$-strongly monotone and $\mathrm{L}_{1}$-Lipschitz continuous, it is easy to see that $\Gamma$ is $v \bar{\eta}+\frac{1}{\xi}$-strongly monotone and Lipschitzian with constant $v \mathrm{~L}_{1}+\frac{1}{\xi}>0$. Thus, there exists a unique solution $x^{*} \in \Omega$ to the VIP

$$
\left\langle v \mathcal{A} x^{*}+\frac{1}{\bar{\xi}}\left(x^{*}-u\right), p-x^{*}\right\rangle \geqslant 0, \quad \forall p \in \Omega
$$

Utilizing the similar arguments to those of (3.22), (3.24), and (3.26), we get

and

$$
\begin{aligned}
& \left\|v_{k}-p\right\|^{2} \leqslant\left\|u_{k}-p\right\|^{2}+2 \alpha_{k} v\left(\left\langle\mathcal{A p}, p-x_{k}\right\rangle+\|\mathcal{A} p\|\left\|x_{k}-u_{k}\right\|\right)+v^{2} \alpha_{k}^{2}\left\|\mathcal{A} u_{k}\right\|^{2}, \\
& \left\|u_{k}-p\right\|^{2} \leqslant\left\|x_{k}-p\right\|^{2}+\lambda_{k} \cdot \frac{\mu^{2}\|F p\|^{2}}{\tau a}
\end{aligned}
$$

$$
\left\|x_{k+1}-p\right\|^{2} \leqslant \beta_{k}\left\|x_{k}-p\right\|^{2}+\gamma_{k}\left\|v_{k}-p\right\|^{2}+\gamma_{k} \bar{\epsilon}_{k}\left(2\left\|z_{k}-p\right\|+\bar{\epsilon}_{k}\right)+2 \alpha_{k}\left\langle u-p, x_{k+1}-p\right\rangle .
$$

Combining the last three inequalities, we get

$$
\begin{aligned}
\left\|x_{k+1}-p\right\|^{2} \leqslant & \beta_{k}\left\|x_{k}-p\right\|^{2}+\gamma_{k}\left[\left\|u_{k}-p\right\|^{2}+2 \alpha_{k} v\left(\left\langle\mathcal{A} p, p-x_{k}\right\rangle+\|\mathcal{A} p\|\left\|x_{k}-u_{k}\right\|\right)\right. \\
& \left.+v^{2} \alpha_{k}^{2}\left\|\mathcal{A} u_{k}\right\|^{2}\right]+\gamma_{k} \bar{\epsilon}_{k}\left(2\left\|z_{k}-p\right\|+\bar{\epsilon}_{k}\right)+2 \alpha_{k}\left\langle u-p, x_{k+1}-p\right\rangle \\
\leqslant & \beta_{k}\left\|x_{k}-p\right\|^{2}+\gamma_{k}\left[\left\|x_{k}-p\right\|^{2}+\lambda_{k} \cdot \frac{\mu^{2}\|F p\|^{2}}{\tau a}+2 \alpha_{k} v\left(\left\langle\mathcal{A} p, p-x_{k}\right\rangle\right.\right. \\
& \left.\left.+\|\mathcal{A} p\|\left\|x_{k}-u_{k}\right\|\right)+v^{2} \alpha_{k}^{2}\left\|\mathcal{A} u_{k}\right\|^{2}\right]+\gamma_{k} \bar{\epsilon}_{k}\left(2\left\|z_{k}-p\right\|+\bar{\epsilon}_{k}\right)+2 \alpha_{k}\left\langle u-p, x_{k+1}-p\right\rangle \\
= & \left(1-\alpha_{k}\right)\left\|x_{k}-p\right\|^{2}+\gamma_{k} \lambda_{k} \cdot \frac{\mu^{2}\|F p\|^{2}}{\tau a}+2 \gamma_{k} \alpha_{k}\left(\left\langle v \mathcal{A} p, p-x_{k}\right\rangle\right. \\
& \left.+\|v \mathcal{A} p\|\left\|x_{k}-u_{k}\right\|\right)+\gamma_{k} \alpha_{k}^{2} v^{2}\left\|\mathcal{A} u_{k}\right\|^{2}+\gamma_{k} \bar{\epsilon}_{k}\left(2\left\|z_{k}-p\right\|+\bar{\epsilon}_{k}\right) d+2 \alpha_{k}\left\langle u-p, x_{k+1}-p\right\rangle \\
\leqslant & \left\|x_{k}-p\right\|^{2}+\gamma_{k} \lambda_{k} \cdot \frac{\mu^{2}\|F p\|^{2}}{\tau a}+2 \gamma_{k} \alpha_{k}\left(\left\langle v \mathcal{A} p+\frac{1}{\gamma_{k}}(p-u), p-x_{k}\right\rangle\right. \\
& \left.+\|v \mathcal{A} p\|\left\|x_{k}-u_{k}\right\|\right)+\gamma_{k} \alpha_{k}^{2} v^{2}\left\|\mathcal{A} u_{k}\right\|^{2}+\gamma_{k} \bar{\epsilon}_{k}\left(2\left\|z_{k}-p\right\|+\bar{\epsilon}_{k}\right) \\
& +2 \alpha_{k}\left\langle u-p, x_{k+1}-x_{k}\right\rangle,
\end{aligned}
$$

which hence implies that

$$
\begin{aligned}
\left\langle v \mathcal{A} p+\frac{1}{\gamma_{k}}(p-u), x_{k}-p\right\rangle \leqslant & \frac{1}{2 \gamma_{k} \alpha_{k}}\left(\left\|x_{k}-p\right\|^{2}-\left\|x_{k+1}-p\right\|^{2}\right)+\frac{\lambda_{k}}{2 \alpha_{k}} \frac{\mu^{2}\|F p\|^{2}}{\tau a} \\
& +\|v \mathcal{A} p\|\left\|x_{k}-u_{k}\right\|+\frac{\alpha_{k} v^{2}}{2}\left\|\mathcal{A} u_{k}\right\|^{2}+\frac{\bar{\epsilon}_{k}}{2 \alpha_{k}}\left(2\left\|z_{k}-p\right\|+\bar{\epsilon}_{k}\right) \\
& +\frac{1}{\gamma_{k}}\left\langle u-p, x_{k+1}-x_{k}\right\rangle \\
\leqslant & \frac{\left\|x_{k}-x_{k+1}\right\|}{2 \gamma_{k} \alpha_{k}}\left(\left\|x_{k}-p\right\|+\left\|x_{k+1}-p\right\|\right)+\frac{\lambda_{k}}{2 \alpha_{k}} \frac{\mu^{2}\|F p\|^{2}}{\tau a} \\
& +\|v \mathcal{A} p\|\left\|x_{k}-u_{k}\right\|+\frac{\alpha_{k} v^{2}}{2}\left\|\mathcal{A} u_{k}\right\|^{2}+\frac{\bar{\epsilon}_{k}}{2 \alpha_{k}}\left(2\left\|z_{k}-p\right\|+\bar{\epsilon}_{k}\right) \\
& +\frac{1}{\gamma_{k}}\|u-p\|\left\|x_{k+1}-x_{k}\right\| .
\end{aligned}
$$

Since for any $w \in \omega_{w}\left(x_{k}\right)$ there exists a subsequence $\left\{x_{k_{i}}\right\}$ of $\left\{x_{k}\right\}$ such that $x_{k_{i}} \rightarrow w$, we deduce from 
(3.34), $\left\|u_{k}-x_{k}\right\| \rightarrow 0$ (due to (3.31)), $\frac{1}{\gamma_{k}} \rightarrow \frac{1}{\bar{\xi}}, \lambda_{k}=o\left(\alpha_{k}\right)$ and $\left\|x_{k+1}-x_{k}\right\|+\bar{\epsilon}_{k}=o\left(\alpha_{k}\right)$ that

$$
\begin{aligned}
\left\langle v \mathcal{A} p+\frac{1}{\bar{\xi}}(p-u), w-p\right\rangle= & \lim _{i \rightarrow \infty}\left\langle v \mathcal{A} p+\frac{1}{\gamma_{k_{i}}}(p-u), x_{k_{i}}-p\right\rangle \\
\leqslant & \limsup _{k \rightarrow \infty}\left\langle v \mathcal{A p}+\frac{1}{\gamma_{k}}(p-u), x_{k}-p\right\rangle \\
\leqslant & \limsup _{k \rightarrow \infty} \frac{\left\|x_{k}-x_{k+1}\right\|}{2 \gamma_{k} \alpha_{k}}\left(\left\|x_{k}-p\right\|+\left\|x_{k+1}-p\right\|\right)+\limsup _{k \rightarrow \infty} \frac{\lambda_{k}}{2 \alpha_{k}} \frac{\mu^{2}\|F p\|^{2}}{\tau a} \\
& +\limsup _{k \rightarrow \infty}\|v \mathcal{A p}\|\left\|x_{k}-u_{k}\right\|+\limsup _{k \rightarrow \infty} \frac{\alpha_{k} v^{2}}{2}\left\|\mathcal{A} u_{k}\right\|^{2} \\
& +\limsup _{k \rightarrow \infty} \frac{\bar{\epsilon}_{k}}{2 \alpha_{k}}\left(2\left\|z_{k}-p\right\|+\bar{\epsilon}_{k}\right)+\limsup _{k \rightarrow \infty} \frac{1}{\gamma_{k}}\|u-p\|\left\|x_{k+1}-x_{k}\right\|=0 .
\end{aligned}
$$

So, it follows that

$$
\left\langle v \mathcal{A} p+\frac{1}{\bar{\xi}}(p-u), p-w\right\rangle \geqslant 0, \quad \forall p \in \Omega .
$$

Since $w \in \omega_{w}\left(x_{k}\right) \subset \Omega$, by Lemma 2.5 (i) (Minty's lemma), we have

$$
\left\langle v \mathcal{A} w+\frac{1}{\bar{\xi}}(w-u), p-w\right\rangle \geqslant 0, \quad \forall p \in \Omega,
$$

that is, $w$ is a solution of VIP (3.32). Utilizing the uniqueness of solutions of VIP (3.32), we get $w=x^{*}$, which hence implies that $\omega_{w}\left(x_{k}\right)=\left\{x^{*}\right\}$. Therefore, it is known that $\left\{x_{k}\right\}$ converges weakly to the unique solution $x^{*} \in \Omega$ of VIP (3.32).

Step 4. We prove that $\left\|x_{k}-x^{*}\right\| \rightarrow 0$ as $k \rightarrow \infty$.

Indeed, utilizing (3.33) with $p=x^{*}$, we get

$$
\begin{aligned}
\left\|x_{k+1}-x^{*}\right\|^{2} \leqslant & \left(1-\alpha_{k}\right)\left\|x_{k}-x^{*}\right\|^{2}+\gamma_{k} \lambda_{k} \frac{\mu^{2}\left\|F x^{*}\right\|^{2}}{\tau a}+2 \gamma_{k} \alpha_{k}\left(\left\langle v \mathcal{A} x^{*}, x^{*}-x_{k}\right\rangle\right. \\
& \left.+\left\|v \mathcal{A} x^{*}\right\|\left\|x_{k}-u_{k}\right\|\right)+\gamma_{k} \alpha_{k}^{2} v^{2}\left\|\mathcal{A} u_{k}\right\|^{2}+\gamma_{k} \bar{\epsilon}_{k}\left(2\left\|z_{k}-x^{*}\right\|+\bar{\epsilon}_{k}\right) \\
& +2 \alpha_{k}\left\langle u-x^{*}, x_{k+1}-x^{*}\right\rangle \\
\leqslant & \left(1-\alpha_{k}\right)\left\|x_{k}-x^{*}\right\|^{2}+\lambda_{k} \frac{\mu^{2}\left\|F x^{*}\right\|^{2}}{\tau a}+2 \gamma_{k} \alpha_{k}\left\langle v \mathcal{A} x^{*}, x^{*}-x_{k}\right\rangle \\
& +2 \alpha_{k}\left\|v \mathcal{A} x^{*}\right\|\left\|x_{k}-u_{k}\right\|+\alpha_{k}^{2} v^{2}\left\|\mathcal{A} u_{k}\right\|^{2}+\bar{\epsilon}_{k}\left(2\left\|z_{k}-x^{*}\right\|+\bar{\epsilon}_{k}\right) \\
& +2 \alpha_{k}\left\langle u-x^{*}, x_{k+1}-x^{*}\right\rangle \\
= & \left(1-\alpha_{k}\right)\left\|x_{k}-x^{*}\right\|^{2}+\alpha_{k}\left[\frac{\lambda_{k}}{\alpha_{k}} \frac{\mu^{2}\left\|F x^{*}\right\|^{2}}{\tau a}+\frac{\bar{\epsilon}_{k}}{\alpha_{k}}\left(2\left\|z_{k}-x^{*}\right\|+\bar{\epsilon}_{k}\right)\right. \\
& +2\left\|v \mathcal{A} x^{*}\right\|\left\|x_{k}-u_{k}\right\|+\alpha_{k} v^{2}\left\|\mathcal{A} u_{k}\right\|^{2} \\
& \left.+2 \gamma_{k}\left\langle v \mathcal{A} x^{*}, x^{*}-x_{k}\right\rangle+2\left\langle u-x^{*}, x_{k+1}-x^{*}\right\rangle\right] .
\end{aligned}
$$

Since $\alpha_{k}+\beta_{k}+\gamma_{k}=1, \alpha_{k} \rightarrow 0, \beta_{k} \rightarrow \xi \in\left(0, \frac{1}{2}\right], \bar{\epsilon}_{k}=o\left(\alpha_{k}\right), \lambda_{k}=o\left(\alpha_{k}\right)$, and $\left\|u_{k}-x_{k}\right\| \rightarrow 0$ (due to (3.31)), we deduce from the boundedness of $\left\{x_{k}\right\},\left\{u_{k}\right\}$, and $\left\{z_{k}\right\}$ that

$$
\begin{aligned}
& \limsup _{k \rightarrow \infty}\left\{\frac{\lambda_{k}}{\alpha_{k}} \frac{\mu^{2}\left\|F x^{*}\right\|^{2}}{\tau a}+\frac{\bar{\epsilon}_{k}}{\alpha_{k}}\left(2\left\|z_{k}-x^{*}\right\|+\bar{\epsilon}_{k}\right)+2\left\|v \mathcal{A} x^{*}\right\|\left\|x_{k}-u_{k}\right\|\right. \\
& \left.+\alpha_{k} v^{2}\left\|\mathcal{A} u_{k}\right\|^{2}+2 \gamma_{k}\left\langle v \mathcal{A} x^{*}, x^{*}-x_{k}\right\rangle+2\left\langle u-x^{*}, x_{k+1}-x^{*}\right\rangle\right\} \leqslant 0 .
\end{aligned}
$$

Consequently, applying Lemma 2.12 to (3.35), we derive

$$
\lim _{k \rightarrow \infty}\left\|x_{k}-x^{*}\right\|=0
$$

This completes the proof. 
It is clear that a mapping $\mathrm{T}: \mathrm{H} \rightarrow \mathrm{H}$ is a $\zeta$-strict pseudocontraction if and only if there exists a constant $\zeta \in[0,1)$ such that

$$
\langle\mathrm{T} x-\mathrm{T} y, x-\mathrm{y}\rangle \leqslant\|x-y\|^{2}-\frac{1-\zeta}{2}\|(\mathrm{I}-\mathrm{T}) \mathrm{x}-(\mathrm{I}-\mathrm{T}) \mathrm{y}\|^{2}, \quad \forall x, y \in \mathrm{H} .
$$

It is easy to see that in this case the mapping $\mathrm{I}-\mathrm{T}$ is $\frac{1-\zeta}{2}$-inverse strongly monotone. According to Lemma 2.7 (i), we know that if $\mathrm{T}$ is a $\zeta$-strictly pseudocontractive mapping, then $\mathrm{T}$ is Lipschitz continuous with constant $\frac{1+\zeta}{1-\zeta}$, i.e., $\|T x-T y\| \leqslant \frac{1+\zeta}{1-\zeta}\|x-y\|$ for all $x, y \in H$. We denote by $\operatorname{Fix}(T)$ the fixed point set of $T$. It is obvious that the class of strict pseudocontractions strictly includes the class of nonexpansive mappings and the class of pseudocontractions strictly includes the class of strict pseudocontractions.

Corollary 3.12. Let $\mathrm{A}=\mathrm{I}-\mathrm{T}$ where $\mathrm{T}$ is a $\zeta$-strict pseudocontraction on $\mathrm{H}$. Let $\beta=\frac{1-\zeta}{2}$. Suppose that the conditions (A1) and (H1)-(H4) hold. Then the two sequences $\left\{x_{k}\right\}$ and $\left\{z_{k}\right\}$ in Algorithm 3.2 converge strongly to the same point $x^{*} \in \Omega:=\operatorname{VI}\left(\mathrm{VI}\left(\cap_{i=1}^{\mathrm{N}} \operatorname{Fix}\left(\mathrm{T}_{i}\right), \mathrm{B}\right), \mathrm{I}-\mathrm{T}\right)$ provided $\left\|\mathrm{x}_{\mathrm{k}}-v_{\mathrm{k}}\right\|=\mathrm{o}\left(\alpha_{\mathrm{k}}\right)$, which is a unique solution to the VIP

$$
\left\langle\mathcal{A} x^{*}, \mathrm{p}-\mathrm{x}^{*}\right\rangle \geqslant 0, \quad \forall \mathrm{p} \in \Omega .
$$

Proof. Putting $A=I-T$ in Theorem 3.10, we know that $A$ is $\beta$-inverse strongly monotone with $\beta=\frac{1-\zeta}{2}$. In this case, we get

$$
\Omega:=\operatorname{VI}\left(\operatorname{VI}\left(\cap_{i=1}^{N} \operatorname{Fix}\left(T_{i}\right), B\right), A\right)=\operatorname{VI}\left(\operatorname{VI}\left(\cap_{i=1}^{N} \operatorname{Fix}\left(T_{i}\right), B\right), I-T\right) .
$$

So, utilizing Theorem 3.10 we derive the desired result.

Corollary 3.13. Let $\mathrm{N}=1$ and $\mathrm{T}$ be a $\zeta$-strict pseudocontraction on $\mathrm{H}$. Let $\mathrm{T}_{1}=\theta \mathrm{I}+(1-\theta) \mathrm{T}$ with $\theta \in[\zeta, 1)$. Suppose that the conditions (A1) and (H1)-(H4) hold. Then the two sequences $\left\{x_{k}\right\}$ and $\left\{z_{k}\right\}$ in Algorithm 3.2 converge strongly to the same point $x^{*} \in \Omega:=\operatorname{VI}(\operatorname{VI}(\operatorname{Fix}(\mathrm{T}), \mathrm{B}), \mathrm{A})$ provided $\left\|x_{\mathrm{k}}-v_{\mathrm{k}}\right\|=\mathrm{o}\left(\alpha_{\mathrm{k}}\right)$, which is a unique solution to the VIP

$$
\left\langle\mathcal{A} x^{*}, \mathrm{p}-\mathrm{x}^{*}\right\rangle \geqslant 0, \quad \forall \mathrm{p} \in \Omega .
$$

Proof. In Theorem 3.10, we put $N=1$ and $T_{1}=\theta \mathrm{I}+(1-\theta) \mathrm{T}$ with $\theta \in[\zeta, 1)$. Since $\mathrm{T}$ is a $\zeta$-strict pseudocontraction on $\mathrm{H}$, it follows from Lemma 2.8 that $\mathrm{T}_{1}$ is a nonexpansive mapping on $\mathrm{H}$. In this case, it is easy to see that $\operatorname{Fix}\left(T_{1}\right)=\operatorname{Fix}(T)$. Thus, we know that

$$
\Omega:=\operatorname{VI}\left(\operatorname{VI}\left(\cap_{i=1}^{N} \operatorname{Fix}\left(T_{i}\right), B\right), A\right)=\operatorname{VI}\left(\operatorname{VI}\left(\operatorname{Fix}\left(T_{1}\right), B\right), A\right)=\operatorname{VI}(\operatorname{VI}(\operatorname{Fix}(T), B), A) .
$$

So, utilizing Theorem 3.10 we obtain the desired result.

\section{Acknowledgment}

This research was partially supported by the National Science Foundation of China (11071169), Innovation Program of Shanghai Municipal Education Commission (09ZZ133) and Ph.D. Program Foundation of Ministry of Education of China (20123127110002). Yeong-Cheng Liou was supported in part by the grand form Kaohsiung Medical University Research Foundation (KMU-Q106005) and Taiwan-Russian joint grant MOST 106-2923-E-039-001-MY3.

\section{References}

[1] P. N. Anh, J. K. Kim, L. D. Muu, An extragradient algorithm for solving bilevel pseudomonotone variational inequalities, J. Global Optim., 52 (2012), 627-639. 1, 1, 1.2, 1.3, 1

[2] L.-C. Ceng, Q. H. Ansari, J.-C. Yao, Iterative methods for triple hierarchical variational inequalities in Hilbert spaces, J. Optim. Theory Appl., 151 (2011), 489-512. 1

[3] L.-C. Ceng, Q. H. Ansari, J.-C. Yao, Relaxed hybrid steepest-descent methods with variable parameters for triplehierarchical variational inequalities, Appl. Anal., 91 (2012), 1793-1810. 2.9 
[4] L.-C. Ceng, C.-T. Pang, C.-F. Wen, Multi-step extragradient method with regularization for triple hierarchical variational inequalities with variational inclusion and split feasibility constraints, J. Inequal. Appl., 2014 (2014), 40 pages. 1

[5] L.-C. Ceng, C.-Y. Wang, J.-C. Yao, Strong convergence theorems by a relaxed extragradient method for a general system of variational inequalities, Math. Methods Oper. Res., 67 (2008), 375-390. 1

[6] L.-C. Ceng, J.-C. Yao, A relaxed extragradient-like method for a generalized mixed equilibrium problem, a general system of generalized equilibria and a fixed point problem, Nonlinear Anal., 72 (2010), 1922-1937. 1

[7] F. Facchinei, J.-S. Pang, Finite-dimensional variational inequalities and complementarity problems, Springer Series in Operations Research, Springer-Verlag, New York, (2003). 1

[8] K. Goebel, W. A. Kirk, Topics in metric fixed point theory, Cambridge Studies in Advanced Mathematics, Cambridge University Press, Cambridge, (1990). 2.6, 2.13

[9] K. Geobel, S. Reich, Uniform convexity, hyperbolic geometry, and nonexpansive mappings, Monographs and Textbooks in Pure and Applied Mathematics, Marcel Dekker, Inc., New York, (1984). i, ii

[10] H. Iiduka, Strong convergence for an iterative method for the triple-hierarchical constrained optimization problem, Nonlinear Anal., 71 (2009), e1292-e1297. 1

[11] H. Iiduka, Iterative algorithm for solving triple-hierarchical constrained optimization problem, J. Optim. Theory Appl., 148 (2011), 580-592. 1, 1.4, 1.5, 1, 1.6, 1

[12] D. Kinderlehrer, G. Stampacchia, An introduction to variational inequalities and their applications, Pure and Applied Mathematics, Academic Press, Inc. [Harcourt Brace Jovanovich, Publishers], New York-London, (1980). i, ii

[13] G. M. Korpelevich, The extragradient method for finding saddle points and other problems, Matecon, 12 (1976), 747-756. 1,1

[14] Z.-Q. Luo, J.-S. Pang, D. Ralph, Mathematical programs with equilibrium constraints, Cambridge University Press, Cambridge, (1996). 1, 1

[15] G. Marino, H.-K. Xu, Weak and strong convergence theorems for strict pseudo-contractions in Hilbert spaces, J. Math. Anal. Appl., 329 (2007), 336-346. 2.7

[16] J. Outrata, M. Kočvara, J. Zowe, Nonsmooth approach to optimization problems with equilibrium constraints, Theory, applications and numerical results, Nonconvex Optimization and its Applications, Kluwer Academic Publishers, Dordrecht, (1998). 1, 1

[17] M. Solodov, An explicit descent method for bilevel convex optimization, J. Convex Anal., 14 (2007), 227-237. 1

[18] T. Suzuki, Strong convergence of Krasnoselskii and Mann's type sequences for one-parameter nonexpansive semigroups without Bochner integrals, J. Math. Anal. Appl., 305 (2005), 227-239. 3.7

[19] V. V. Vasin, A. L. Ageev, Ill-posed problems with a priori information, Inverse and Ill-posed Problems Series, VSP, Utrecht, (1995). iii, iv

[20] H.-K. Xu, Iterative algorithms for nonlinear operators, J. London Math. Soc., 66 (2002), 240-256. 2.12

[21] H.-K. Xu, T. H. Kim, Convergence of hybrid steepest-descent methods for variational inequalities, J. Optim. Theory Appl., 119 (2003), 185-201. 2.10

[22] I. Yamada, The hybrid steepest descent method for the variational inequality problem over the intersection of fixed point sets of nonexpansive mappings, Inherently parallel algorithms in feasibility and optimization and their applications, Haifa, (2000), Stud. Comput. Math., North-Holland, Amsterdam, 8 (2001), 473-504. 1

[23] Y.-H. Yao, R.-D. Chen, H.-K. Xu, Schemes for finding minimum-norm solutions of variational inequalities, Nonlinear Anal., 72 (2010), 3447-3456. 2.1

[24] Y.-H. Yao, Y.-C. Liou, S. M. Kang, Approach to common elements of variational inequality problems and fixed point problems via a relaxed extragradient method, Comput. Math. Appl., 59 (2010), 3472-3480. 1, 2.8

[25] Y.-H. Yao, Y.-C. Liou, J.-C. Yao, An extragradient method for fixed point problems and variational inequality problems, J. Inequal. Appl., 2007 (2007), 12 pages. 3

[26] Y.-H. Yao, M. A. Noor, Y.-C. Liou, Strong convergence of a modified extragradient method to the minimum-norm solution of variational inequalities, Abstr. Appl. Anal., 2012 (2012), 9 pages. 1

[27] Y.-H. Yao, M. A. Noor, Y.-C. Liou, S. M. Kang, Iterative algorithms for general multivalued variational inequalities, Abstr. Appl. Anal., 2012 (2012), 10 pages. 1

[28] L.-C. Zeng, M.-M. Wong, J.-C. Yao, Strong convergence of relaxed hybrid steepest-descent methods for triple hierarchical constrained optimization, Fixed Point Theory Appl., 2012 (2012), 24 pages. 1, 1, 1.7, 1.8, 1, 1.9, 1, 1.10, 1.11, 1

[29] L.-C. Zeng, J.-C. Yao, Implicit iteration scheme with perturbed mapping for common fixed points of a finite family of nonexpansive mappings, Nonlinear Anal., 64 (2006), 2507-2515. 1 